\title{
Wie erreicht man das „gute ökologische Potenzial“? Fallbeispiel Innstauraum Egglfing-Obernberg
}

\author{
Gerald Zauner · Wolfgang Lauber · Michael Jung • Clemens Ratschan · Michael Schöfbenker · Roland Schmalfuß
}

Online publiziert: 6. Mai 2020

(c) Der/die Autor(en) 2020

\begin{abstract}
Zusammenfassung Die Wasserkörper der bayerisch-oberösterreichischen Grenzstrecke des Unteren Inns zwischen dem Kraftwerk Passau-Ingling und der Salzachmündung wurden als „erheblich veränderte Wasserkörper“ entsprechend der EU-Wasserrahmenrichtlinie eingestuft. Hier gilt nicht der „gute ökologische Zustand“, sondern das „gute ökologische Potenzial“ als $\mathrm{zu}$ erreichender Zielzustand. Für die Erreichung dieses Ziels ist ein gemäß „Leitfaden zur Bewertung erheblich veränderter Gewässer" definierter Teil des „höchsten ökologischen Potenzials“ umzusetzen, worunter das maximale Maßnahmenpotenzial ohne signifikante Einschränkungen der für die Einstufung als „erheblich veränderter Wasserkörper" verantwortlichen Nutzungen zu verstehen ist. Für den Innstauraum Egglfing-Obernberg wurden die dazu erforderlichen Maßnahmen definiert und im Rahmen des von 2017 bis 2019

Fehlen hochwertiger Laich- und Juvenilhabitate rheophiler Flussfischarten und die stark eingeschränkte laterale Konnektivität, was sich in quantitativ geringen Fischbeständen und einem Defizit an Arten und Altersstadien äußerte. Allerdings konnte im Zuge dieser Erhebungen mit dem Steingreßling eine im gesamten Salzach-Innsystem ausgestorben geglaubte Fischart wiederentdeckt werden. Erste Untersuchungsergebnisse der herbstlichen Fischmigration im Umgehungsgewässer zeigten ungewöhnlich hohe Aufstiegszahlen verschiedener Cyprinidenarten. Im Rahmen des über die nächsten Jahre laufenden Monitorings soll die Wirkung der Maßnahmen in Hinblick auf die Zielerreichung nach Wasserrahmenrichtlinie untersucht werden, wobei in Analogie zu den Ergebnissen bestehender fischökologischer Studien eine deutliche Stärkung der Fischpopulationen zu erwarten ist.
\end{abstract} laufenden Projekts „Durchgängigkeit und Lebensraum" zum Großteil bereits umgesetzt. Projektinhalte waren die Herstellung eines Insel-Nebenarmsystems einschließlich eines unterstromig angebundenen Stillgewässers in der Stauwurzel, die Dynamisierung und Entlandung von bestehenden Altarmen sowie die Errichtung eines naturnahen Umgehungsgewässers als Fischaufstiegshilfe und hochwertiger Fließgewässerlebensraum. Fischökologische Erhebungen vor Maßnahmenumsetzung bestätigten die hydromorphologischen Defizite des Innstauraums Egglfing-Obernberg, insbesondere das

DI Dr. G. Zauner $(\bowtie) \cdot$ DI W. Lauber • Mag. M. Jung • Mag. C. Ratschan . M. Schöfbenker, MSc. ezb - TB Zauner GmbH, Marktstraße 35, 4090 Engelhartszell, Österreich

zauner@ezb-fluss.at

DI Dr. R. Schmalfuß

VERBUND Hydro Power GmbH, Europaplatz 2, 1150 Wien, Österreich
Schlüsselwörter EU-

Wasserrahmenrichtlinie - Erheblich veränderter Wasserkörper · HMWB . Ökologisches Potenzial .

Gewässerrenaturierung · Naturnahe Fischaufstiegshilfe .

Umgehungsgerinne $\cdot$ Nebenarm

How to achieve the "good ecological potential" according to the EU Water Framework Directive? A case study from the Inn impoundment EgglfingObernberg

Abstract According to the EU water framework directive, the water bodies of the Lower Inn at the Bavarian-Austrian border between the hydropower plant Passau-Ingling and the Salzach river mouth are classified as "heavily modified water bodies" (HWMB). Therefore, not a "good ecological status" but a "good ecological potential" has to be achieved. "heavily modified water body guide-
In accordance with the Austrian line", a defined amount of measures of the "maximum ecological potential" has to be implemented to achieve the "good ecological potential". The "maximum ecological potential" includes all potential mitigation measures that do not have significant adverse effects on the HWMB specified water use. Mitigation measures have been developed for the Inn impoundment Egglfing-Obernberg and most of them have been implemented as part of the project "Durchgängigkeit und Lebensraum" ("Connectivity and habitat") between 2017 and 2019. The project contained the construction of a new side branch and an oxbow, the desedimentation and activation of existing oxbows and the construction of a nature-like bypass channel serving as fishpass as well as valuable fluvial habitat.

Preceding fish ecological surveys have confirmed the hydromorphological deficits of the Inn impoundment Egglfing-Obernberg. In particular, a lack of spawning and juvenile habitats for rheophilic fish species and a strongly limited lateral connectivity can be emphasized. These deficits result in a low fish stock and the absence of species and age classes. Nevertheless, the endangered stone gudgeon has been rediscovered for the InnSalzach river system. First results of the monitoring of the fish migration in the nature-like bypass during last autumn indicated an unusually high number of migrating fish, especially subadult Cyprinids. Within the ongoing monitoring over the next couple of years, the impact of the mitigation measures in response to the objectives of the EU water framework directive will be examined. The authors expect comparably positive effects, based on existing fish ecological studies from the Danube river, which have indicated significant improvements of riverine fish populations. 
Keywords EU water framework directive $\cdot$ Heavily modified water body - Ecological potential - River restoration - Nature-like fishway · Bypass channel · Side branch

\section{Einleitung}

Mit der im Jahre 2000 in Kraft getretenen EU-Wasserrahmenrichtlinie (WRRL) wurden neue Maßstäbe in der europäischen Gewässerschutzpolitik gesetzt. Ziel der Richtlinie ist es, den „guten ökologischen Zustand“ aller Gewässer (Seen, Fließgewässer und Grundwasser) zu erhalten bzw. innerhalb eines bestimmten Zeitraums wiederherzustellen. Bei durch menschliche Nutzungen wie beispielsweise Wasserkraft stark veränderten Gewässern ("heavily modified water bodies“) wie dem Unteren Inn ist dabei das "gute ökologische Potenzial“ zu erreichen.

An den österreichischen Fließgewässern im Allgemeinen und am Unteren Inn im Speziellen stellen derzeit hydromorphologische Belastungen das Hauptdefizit dar (BMLFUW 2017). Daher kommt der Herstellung und Aufwertung fließgewässertypischer Lebensräume prioritäre Bedeutung für die Erreichung des „guten ökologischen $\mathrm{Zu}$ standes/Potenzials“ zu. Gemäß Nationalem Gewässerbewirtschaftungsplan weist der Stauraum Egglfing-Obernberg (Wasserkörper 305340007) - wie die gesamte Staukette des Unteren Inn - derzeit ein „mäßiges oder schlechteres Potenzial“ auf (BMLUFW 2017). Für diese Einstufung zeichnen primär die defizitären fischökologischen Verhältnisse verantwortlich, welche die weitreichenden hydromorphologischen Belastungen widerspiegeln. Gerade für die Hydromorphologie ist die Fischzönose das maßgebliche biologische Qualitätselement.

Innwerk AG, eine Gesellschaft von VERBUND, ist Konsensträgerin der Innkraftwerke Ering-Frauenstein und Egglfing-Obernberg an der bayerischoberösterreichischen Grenze. Die Anlagen wurden während des 2. Weltkriegs bewilligt und errichtet. In den letzten Jahren beantragte Innwerk AG in Österreich und Deutschland die Wiederverleihung der Wasserrechte bzw. die Bewilligungen des Weiterbetriebs der Kraftwerksanlagen. Zur umfassenden Wiederherstellung der flussauf gerichteten Durchgängigkeit und zur Förderung der Fischpopulationen in den Innstauräumen durch Schaffung von Lebensraum und Verbesserung des Fluss-Ausystems plant und errichtet Innwerk AG an beiden Kraftwerken dynamisch dotierte Umgehungsgewässer und Unterwasserstrukturierungen. Zusammen mit weiteren Projekten zur Schaffung von Gewässerlebensraum in den Stauräumen und an Zubringermündungen stellen diese Maßnahmen einen wesentlichen Beitrag zur Erreichung des "guten ökologischen Potenzials“ in den Detailwasserkörpern des Grenzinns dar.

\section{Der Weg zum ,guten}

ökologischen Potenzial" in einer Staukette gemäß Leitfaden

Für erheblich veränderte und künstliche Wasserkörper ist anstelle des "guten ökologischen Zustandes" ein abweichendes Güteziel, nämlich das "gute ökologische Potenzial“ als Zielzustand zu erhalten bzw. zu erreichen. Die Staukette des Unteren Inns bzw. die einzelnen Detailwasserkörper wurden als erheblich veränderte Wasserkörper eingestuft. Demnach orientieren sich die ökologische Bewertung und die sich daraus ergebenden Maßnahmenpläne nicht - wie bei natürlichen Gewässern - am „sehr guten Zustand“ als Bezugsmaßstab (Referenzzustand), sondern am „höchsten ökologischen Potenzial“. Das „höchste ökologische Potenzial“ ist ,jener Zustand der Gewässerbiozönose, der unter den für die Ausweisung als erheblich verändertes Gewässer verantwortlichen Rahmenbedingungen (d.h. alle technisch möglichen Maßnahmen, die die Nutzung(en) oder die weitere Umwelt nicht signifikant gefährden) möglich ist" (Eberstaller et al. 2015).

Als Zielvorgabe bzw. Zielzustand wird in der WRRL für diese Gewässer das "gute ökologische Potenzial“ verankert, das eine geringe Abweichung vom „höchsten ökologischen Potenzial“ darstellt. Ist die Abweichung vom „höchsten ökologischen Potenzial" mehr als nur gering, dann ist ein „mäßiges“, „unbefriedigendes“ oder „schlechtes ökologisches Potenzial“ gegeben und die Mitgliedstaaten müssen Sanierungsmaßnahmen zur Erreichung der Zielvorgabe setzen. Wie bereits erwähnt, trifft dies auf die Detailwasserkörper des Unteren Inns zwischen der Salzachmündung und dem Kraftwerk Passau-Ingling zu.

Die Definition des ökologischen Potenzials erfolgt dabei - wie bei den natürlichen Wasserkörpern - grundsätzlich über den Zustand der Gewässerzönose. Das bedeutet, dass die Bewertung auf Basis einer Analyse der biologischen Qualitätselemente (Phytobenthos, Makrophyten, Makrozoobenthos, Fische) und dem Vergleich des Istzustands mit dem Sollzustand („höchstes ökologisches Potenzial“) zu erfolgen hat. Mangels biologischer Daten und Erfahrungen, die eine exakte Ableitung des ökologischen Potenzials anhand von biologischen Kennwerten ermöglichen, wurde im „Leitfaden zur Bewertung erheblich veränderter $\mathrm{Ge}$ wässer“ (nachfolgend kurz „Leitfaden“; Eberstaller et al. 2015) ein praktikabler Alternativansatz vorgeschlagen. Dabei wird das ökologische Potenzial im Wesentlichen über jene Verbesserungsmaßnahmen definiert, die zu einer deutlichen ökologischen Verbesserung führen und keine signifikanten Auswirkungen auf die Nutzung haben. Die Maßnahmen müssen allerdings auf ein biologisches Ziel ausgerichtet sein, das sich aus der Annäherung an jenen natürlichen Gewässertyp ergibt, der dem veränderten Gewässertyp am ehesten entspricht.

Für die Festlegung des ökologischen Potenzials ist im ersten Schritt eine Kombination aus den beiden beschriebenen Vorgangsweisen anzuwenden. Die Definition des ökologischen Potenzials erfolgt durch eine verbale Beschreibung des fischökologischen Zustands und wird durch eine maßnahmenorientierte Beschreibung der jeweiligen hydromorphologischen Eigenschaften ergänzt. Durch die biologische Beschreibung des Zustands wird die Zielrichtung der gewählten Maßnahmen (z.B. Erhalt eigenständiger Bestände für einen wesentlichen Teil der Fischfauna) vorgegeben.

Im Leitfaden werden die dafür notwendigen Bearbeitungsschritte aufgezeigt und es wird ein Überblick über mögliche Maßnahmen und deren Verbesserungspotenzial gegeben. Folgt man diesem Konzept sind folgende Bearbeitungsschritte notwendig:

- Festlegung der im jeweiligen Gewässerabschnitt/Wasserkörper technisch möglichen Maßnahmen, die keine signifikante Beeinträchtigung der Nutzung zur Folge haben.

- Abschätzung der entstehenden Lebensraumverhältnisse und der dadurch resultierenden Verbesserungen für charakteristische Gruppen 


\begin{tabular}{|c|c|c|c|c|c|c|c|c|c|c|c|c|c|c|c|c|c|}
\hline \multirow{3}{*}{$\begin{array}{l}\text { Maßnahmentyp } \\
\text { k1. Strukturierung SW }\end{array}$} & \multicolumn{9}{|c|}{ Rheophile + kieslaichende Indifferente } & \multicolumn{8}{|c|}{ Indifferente + Stagnophile } \\
\hline & \multicolumn{3}{|c|}{ Reproduktion } & \multicolumn{3}{|c|}{$\begin{array}{l}\text { Lebensraum } \\
\text { Juvenile }\end{array}$} & \multicolumn{3}{|c|}{$\begin{array}{l}\text { Lebensraum } \\
\text { Adulte }\end{array}$} & \multicolumn{3}{|c|}{ Reproduktion } & \multicolumn{3}{|c|}{$\begin{array}{c}\text { Lebensraum } \\
\text { Juvenile }\end{array}$} & \multicolumn{2}{|c|}{$\begin{array}{l}\text { Lebensraum } \\
\text { Adulte }\end{array}$} \\
\hline & ++ & bis & +H & ++ & bis & ++ & + & bis & + & ++ & bis & +++ & ++ & bis & +++ & bis & ++ \\
\hline gr. Strukturierung SW & +++ & bis & H+r & +H & bis & +r+t & ++ & bis & +r+ & ++ & bis & ++ & +++ & bis & t+r+ & $+\quad$ bis & ++ \\
\hline $\begin{array}{l}\text { kl. Umgehungsgerinne } \\
\text { (nur Lebensraum) }\end{array}$ & ++ & bis & +H & + & bis & +++ & + & bis & ++ & + & bis & ++ & + & bis & ++ & bis & ++ \\
\hline $\begin{array}{l}\text { gr. Umgehungsgerinne } \\
\text { (nur Lebensraum) }\end{array}$ & +++ & bis & +H++ & ++t & bis & +t++ & ++ & bis & +++ & ++ & bis & +++ & ++ & bis & +t++ & $+\quad$ bis & +++ \\
\hline $\begin{array}{l}\text { Strukturierung Ufer } \\
\text { zentr. Stau }\end{array}$ & \multicolumn{3}{|c|}{ bis } & + & bis & +++ & & bis & + & + & bis & +++ & + & bis & +++ & bis & +H+ \\
\hline Flachwasserzonen & \multicolumn{3}{|c|}{ bis } & + & bis & ++ & & bis & & & bis & +r+ & ++ & bis & +r+t & $+\quad$ bis & ++ \\
\hline $\begin{array}{l}\text { Anlage/Vernetzung } \\
\text { Nebengewässer }\end{array}$ & \multicolumn{3}{|c|}{ bis } & + & bis & ++ & & bis & + & ++ & bis & +h++ & ++ & bis & tat+ & $++\quad$ bis & +H+ \\
\hline $\begin{array}{l}\text { Vernetzung intakter } \\
\text { Zuflüsse }\end{array}$ & ++ & bis & +h++ & ++ & bis & +++ & + & bis & ++ & + & bis & ++ & + & bis & ++ & bis & ++ \\
\hline $\begin{array}{l}\text { Vernetzung mit gutem } \\
\text { Lebensraum (gut strukt. } \\
\text { SW) }\end{array}$ & ++ & bis & +++ & ++ & bis & +++ & ++ & bis & ++ & & bis & +++ & +++ & bis & +t+t+ & $++\quad$ bis & +++ \\
\hline $\begin{array}{l}\text { Vernetzung mit } \\
\text { schlechtem Lebensraum } \\
\text { (Stau) }\end{array}$ & + & bis & ++ & & bis & ++ & + & bis & ++ & + & bis & +++ & ++ & bis & +++ & $++\quad$ bis & +++ \\
\hline $\begin{array}{l}\text { Vernetzung mit gr. } \\
\text { Vorfluter }\end{array}$ & + & bis & +++ & + & bis & +++ & + & bis & +++ & ++ & bis & +++ & ++ & bis & +++ & ++ bis & +++ \\
\hline $\begin{array}{l}\text { Erhöhung Kieseintrag } \\
\text { aus OW }\end{array}$ & + & bis & ++ & + & bis & ++ & + & bis & ++ & + & bis & ++ & & bis & ++ & bis & ++ \\
\hline $\begin{array}{l}\text { ökolog. optimierter } \\
\text { Feinsedimenteintrag aus } \\
\text { OW }\end{array}$ & + & bis & ++ & + & bis & ++ & + & bis & ++ & + & bis & ++ & & bis & ++ & bis & ++ \\
\hline
\end{tabular}

Abb. 1 Wirkungen unterschiedlicher Maßnahmen in Stauketten auf Gilden und Lebensstadien von Fischen (aus Eberstaller et al. 2015). SW Stauwurzel, OW Oberwasser

der gewässertypischen Fischbestände.

- Festlegen der zulässigen, geringfügigen Abweichung von den biologischen Verhältnissen beim „höchsten ökologischen Potenzial“.

- Auswahl der Maßnahmenkombination, mit der das "gute ökologische Potenzial“" am effizientesten erreicht werden kann.

Bei Stauketten - wie am Unteren Inn - soll das „gute ökologische Potenzial“ primär durch Schaffung und Vernetzung von Gewässerlebensraum erzielt werden. Das bedeutet nach Eberstaller et al. (2015), dass „Wanderungsmöglichkeiten für die Fischfauna sowohl im Fluss als auch in Zuflüsse und Nebengewässer sowie gut strukturierte Stauwurzeln vorhanden sind“. Geeignete Maßnahmen sind die Wiederherstellung der Durchgängigkeit, Anbindung von $\mathrm{Zu}$ flüssen und Nebengewässern sowie die Strukturierung der Stauwurzeln.

Für Stauketten sind die ökologischen Wirkungen $(=$ erreichbare Verbesse- rungen) verschiedener Maßnahmen in Abb. 1 angeführt.

Die biologische Wirkung der einzelnen Maßnahmen auf die insgesamt sechs Aspekte wird im Leitfaden mithilfe des folgenden 5-stufigen Schemas bewertet:

$+\quad$ Geringer Beitrag zur Erfüllung eines Aspekts

++ $\quad$ Mittlerer Beitrag zur Erfüllung eines Aspekts

+++ Starker Beitrag zur Erfüllung eines Aspekts, erfüllt alleine Mindesterfordernis für kurz-/ mittelfristige Erhaltung der Populationen

++++ Sehr starker Beitrag zur Erfüllung eines Aspekts, erfüllt alleine Erfordernis für langfristige Erhaltung der Populationen

+++++ Maßnahme beseitigt fast Defizit, dieser Aspekt verhindert nicht mehr Erreichung des guten Zustandes

Für die Gesamtbewertung eines Aspekts (z.B. Reproduktion rheophi- ler und kieslaichender indifferenter Arten; Lebensraum adulter indifferenter und stagnophiler Arten) werden anschließend die Einzelbewertungen für die einzelnen Maßnahmen gemäß folgender Kombinationsregeln zusammengefasst:

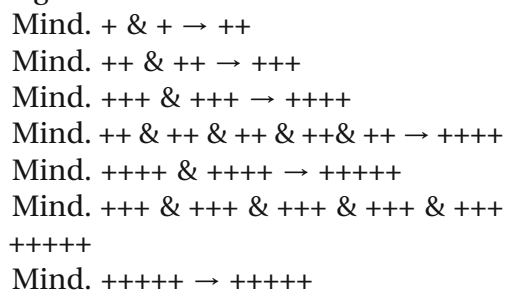

Zur Bestimmung des „höchsten ökologischen Potenzials" werden alle technisch möglichen Maßnahmen, welche die Nutzung nicht signifikant gefährden, ausgewählt und die sich insgesamt ergebende Verbesserung entsprechend dieses Schemas bewertet. Diese Gesamtbewertung für die einzelnen Teilaspekte stellt das „höchste ökologische Potenzial“ dar. 
Das „gute ökologische Potenzial“ wird wie folgt festgelegt:

Werden beim „höchsten Potenzial“ für Teilaspekte starke Verbesserungen erreicht $(++++$ bis +++++$)$, können die bei diesen Teilaspekten erforderlichen Verbesserungen beim ,guten ökologischen Potenzial" um bis zu eine Stufe reduziert werden (z. B.: +++++ $\rightarrow++++$ ). Kann beim „höchsten ökologischen Potenzial“ für Teilaspekte gerade noch etwas mehr als das biologische Mindesterfordernis oder sogar weniger erreicht werden (max. Bewertung $+++(+)$ ), dann weicht das "gute ökologische Potenzial“ nur geringfügig vom „höchsten ökologischen Potenzial“ ab, d.h. um weniger als eine ganze Klasse (z. B.: $+++(+) \rightarrow$ +++ .

\section{Maßnahmenpotenzial zur Zielerreichung am Unteren Inn}

Das ökologische Restrukturierungspotenzial der Innstaustufen entlang der Grenzstrecke zwischen Oberösterreich und Bayern wurde bereits vor 10 Jahren im Rahmen einer Maßnahmenpotenzialstudie ermittelt (Mühlbauer et al. 2011). In dieser Arbeit wird das höchste ökologische Maßnahmenpotenzial im Sinne der WRRL dargestellt. Für den Stauraum Egglfing-Obernberg (DWK 305340007) werden Strukturierungsmaßnahmen im zentralen Stauraum, Zubringerstrukturierungen, Aufweitungsmaßnahmen in der Stauwurzel und dynamische Umgehungsgewässer beschrieben und deren ökologische Wirksamkeit abgeschätzt.

Aufbauend auf den Erkenntnissen dieser Maßnahmenpotenzialstudie wurde ein Konzept zur Ermittlung des „guten ökologischen Potenzials“ im Sinne der Wasserrahmenrichtlinie sowie eine detaillierte Darstellung von konkreten Strukturierungen zur Zielerreichung erarbeitet (Zauner et al. 2015a). Das Bearbeitungsgebiet umfasste die Innstauräume Kraftwerk EgglfingObernberg (Fluss-km 35,50 bis 48,00; österr. DWK 305340007) und Kraftwerk Ering-Frauenstein (Fluss-km 48,00 bis 61,00; österr. DWK 305340009). Der Abschnitt liegt zur Gänze im deutschen Flusswasserkörper IN 153 (Inn von der Einmündung Salzach bis Ingling). Die Lage der beiden Innkraftwerke ist in Abb. 2 dargestellt. In dieser Arbeit wurde für die genannten Wasserkörper eine detaillierte Bilanzierung des Umfangs der Maßnahmen des „höchsten ökologischen Potenzials“ durchgeführt, um

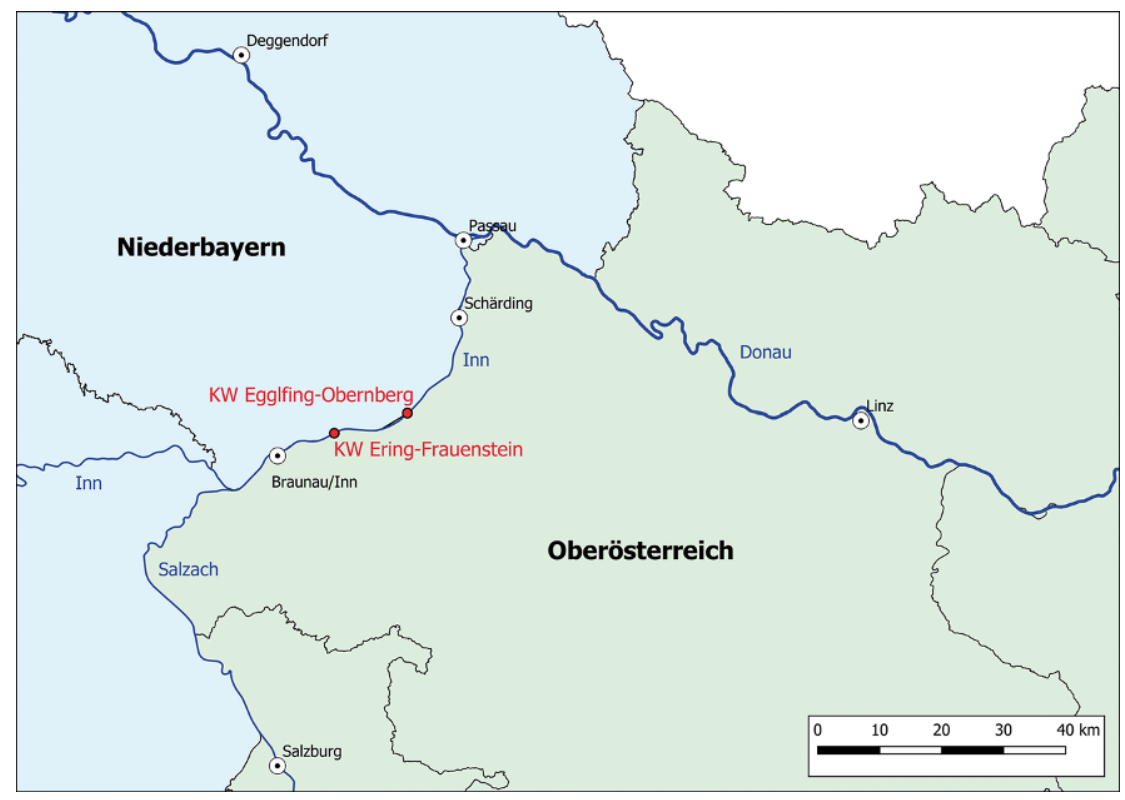

Abb. 2 Lage der beiden Innkraftwerke Ering-Frauenstein und Egglfing-Obernberg. GIS-Datengrundlagen: data.umweltbundesamt.at; Bayerische Vermessungsverwaltung - www.geodaten.bayern. de und Bayerisches Staatsministerium des Innern, für Bau und Verkehr

den Maßnahmenumfang des „guten ökologischen Potenzials“ im Sinne des oben vorgestellten Leitfadens ermitteln zu können. Die dabei angewandte Bewertungsmethode baut auf dem Prinzip auf, dass für die verschiedenen Uferstrukturtypen verschiedene fischökologische Aspekte (Schlüsselhabitatfunktionen, Lebensraumaspekte jeweils für die Gilden „rheophile“ und „indifferente und stagnophile") qualitativ zu bewerten sind. Durch Multiplikation dieser qualitativen Bewertung mit der Länge bzw. Fläche einer Uferstruktur kann die Bedeutung derselben für einen Lebensraumaspekt quantifiziert werden. Dabei erfolgt eine qualitative Bewertung eines Habitattyps durch Abschätzung der relativen Funktionserfüllung in Bezug zu einem natürlichen Referenzhabitat.

Aus einer zusammenfassenden Bewertung ist ableitbar, dass der Schwerpunkt des Potenzials zur Wiederherstellung von Schlüssellebensräumen durch gewässerökologische Strukturmaßnahmen in der Stauwurzel und in dynamischen Umgehungsgewässern liegt. Des Weiteren ist erkennbar, dass auch die Altarm- und Feindsedimentstrukturen des Staus von großer Bedeutung für die indifferente Fischfauna sind. Diese Strukturen sind vielfach im Bestand noch gegeben, weshalb diesbezüglich der Schwerpunkt auf deren Erhalt liegt.
Die ermittelten Maßnahmen lassen sich im Sinne des Leitfadens den Maßnahmentypen

- große Strukturierung Stauwurzel,

- großes Umgehungsgerinne (nur Lebensraum) und

- Anlage/Vernetzung Nebengewässer

zuordnen. Es sind jedoch mehrere Einschränkungen gegenüber dem höchsten Maßnahmenpotenzial gegeben. So sind $\mathrm{u}$. a. aufgrund des bereits bei Mittelwasser ausgeprägten Staucharakters auch in den Stauwurzeln bei der „Reproduktion für Rheophile+kieslaichende Indifferente“ Einschränkungen bezüglich der Wirksamkeit der Stauwurzelstrukturen zu erwarten. Trotz diverser Einschränkungen bei den einzelnen Maßnahmenwirkungen wird gemäß der oben beschriebenen Kombinationsregeln bei Umsetzung aller Maßnahmentypen das „gute ökologischen Potenzial“ erreicht und es ist $\mathrm{zu}$ erwarten, dass dies nach einer gewissen Zeit auch biologisch anhand des Qualitätselements Fische $\mathrm{zu}$ messen sein wird.

Aufbauend auf den Erkenntnissen der Bewertungen wurde seitens VERBUND u. a. das Projekt „Innkraftwerk Ering-Frauenstein - Durchgängigkeit und Lebensraum" in Angriff genommen, welches die zentralen Module zur 
Erreichung des „guten ökologischen Potenzials" beinhaltet.

\section{Projekt „Innkraftwerk Ering- Frauenstein - Durchgängigkeit und Lebensraum"}

Das von 2017 bis 2019 umgesetzte Projekt „Innkraftwerk Ering-Frauenstein Durchgängigkeit und Lebensraum“ beinhaltet umfangreiche Maßnahmen zur Wiederherstellung der flussaufgerichteten Fischdurchgängigkeit am Innkraftwerk Ering-Frauenstein und Verbesserung des Gewässerlebensraums im flussab angrenzenden Innstauraum Egglfing-Obernberg. Dabei handelt es sich im Wesentlichen um die Herstellung eines dynamischen Umgehungsgewässers, eines Insel-Nebenarmsystems in der Stauwurzel des Innkraftwerkes Egglfing-Obernberg sowie die Vernetzung von Nebengewässern einschließlich Dynamisierung der „fossilen $\mathrm{Au}$ “.

\subsection{Dynamisches Umgehungsgewässer}

Das neu errichtete Fließgewässer umgeht das Innkraftwerk Ering-Frauenstein und entspricht funktionell einem kleinen Nebenarm des Inns mit dem Gefälle eines steileren Zubringers (Abb. 3). Aufgrund der naturnahen Ausführung ermöglicht es Fischen nicht nur flussaufwärts zu wandern, sondern bietet auch wertvolle Gewässerlebens-

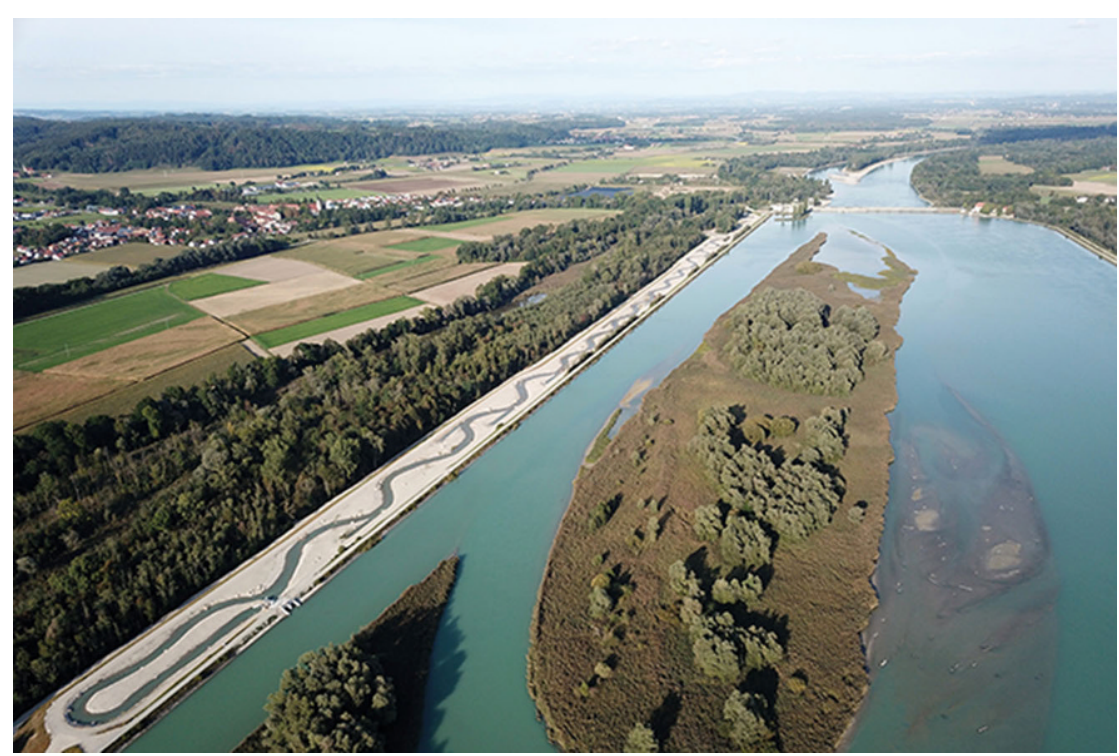

Abb. 3 Umgehungsgewässer bei rund $3 \mathrm{~m}^{3} / \mathrm{s}$ Abfluss mit Blick vom Ausstieg (Dotationsbauwerk) flussab Richtung Kraftwerk Ering-Frauenstein. Im Vordergrund die für die zentralen Stauräume des Unteren Inns typischen, mit Röhrichten und Auwald bewachsenen Feinsedimentinseln

räume wie Kieslaichplätze und Jungfischhabitate.

Auf einer Gesamtlänge von rund 2,6 km überwindet das Umgehungsgewässer eine Höhendifferenz von rund $10 \mathrm{~m}$ zwischen Ober- und Unterwasser. Das mittlere Gefälle beträgt auf den oberen $2 \mathrm{~km} 4,7 \%$ und auf den unteren $600 \mathrm{~m}$ bis ins Unterwasser des Kraftwerks 1,1 \%o. Der Ausstieg bzw. die Dotation des Umgehungsgewässers befindet sich 1,7 km flussauf des Kraftwerks im zentralen Stau, wo keine bzw. nur minimale Wasserspiegelschwankungen auftreten.

Die oberen $250 \mathrm{~m}$ des Gerinnes sind als Schleife („Einlaufgerinne“) mit einem Basisabfluss von $2 \mathrm{~m}^{3} / \mathrm{s}$ ausgeführt. Unterhalb der Schleife erfolgt eine variable (dynamische) Zusatzdotation mit bis $\mathrm{zu} 12 \mathrm{~m}^{3} / \mathrm{s}$ Gesamtabfluss. Die Dotation erfolgt über ein zweigeteiltes Betonbauwerk, das in den Stauhaltungsdamm integriert ist. Der Regelabfluss bewegt sich zwischen 2 und $8 \mathrm{~m}^{3} / \mathrm{s}$ und korreliert mit den mittleren Abflüssen des Inns im Jahresverlauf, wobei die Dotationsmengen hinsichtlich Auffindbarkeit des Einstiegs und Reproduktion von Fischen im Gerinne am Anfang der Betriebsphase optimiert werden sollen.

Zur Überwindung des Höhenunterschieds wurde an der landseitigen Dammböschung des bestehenden InnStauhaltungsdammes auf rund $1,5 \mathrm{~km}$ Länge eine Anschüttung aus im Zuge werke mit Schützen verschließbar ausgeführt wurden, um bei Innhochwässern schutzwasserwirtschaftliche Beeinträchtigungen für das Hinterland zu vermeiden. Im Hochwasserfall $\left(a b H Q_{1}\right)$ wird der Abfluss im Umgehungsgewässer auf die Basisdotation von $2 \mathrm{~m}^{3} / \mathrm{s}$ reduziert und über eine Überströmstrecke in ein bestehendes Gewässer (Kirnbach) sowie einen Durchlass am Kraftwerk vorbei in das Unterwasser geleitet.

Etwa $250 \mathrm{~m}$ flussab des Kraftwerks mündet das Umgehungsgewässer in den Inn und schließt dort unmittelbar an das flussab liegende Insel-Nebenarmsystem an. Zur Optimierung der Uferanströmung und somit der Auffindbarkeit des Umgehungsgewässers wurde ein großzügiger Uferrückbau durchgeführt (Abb. 6). In Kombination mit hohen Abflüssen im Umgehungsgewässer kann dadurch die Auffindbarkeit sichergestellt werden. 


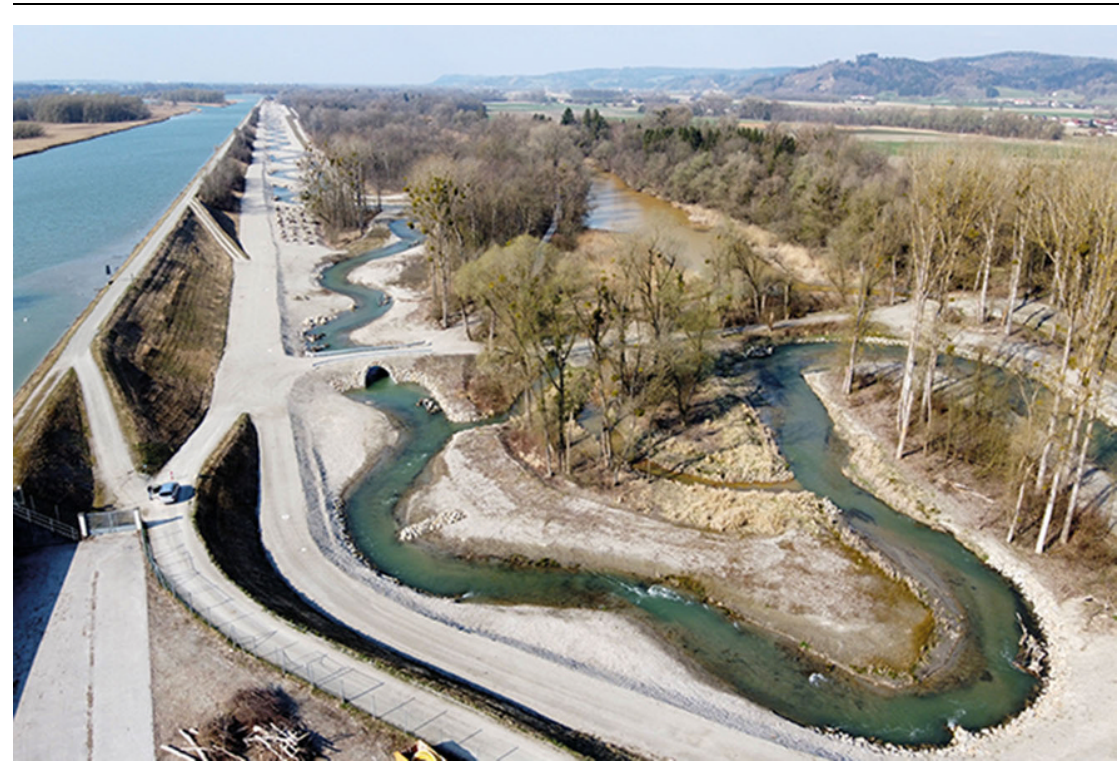

Abb. 5 Verlauf des Umgehungsgewässers im Aueniveau flussab der Anschüttung

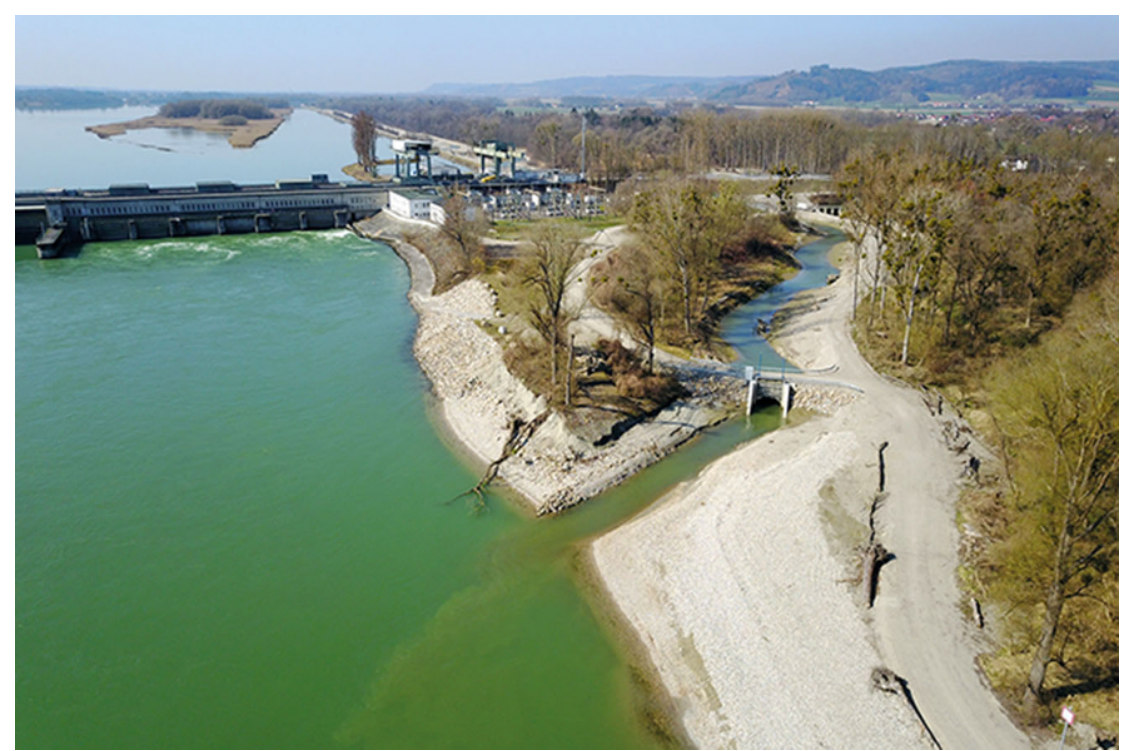

Abb. 6 Der Einstieg (Mündung) in das Umgehungsgewässer befindet sich linksufrig etwa $250 \mathrm{~m}$ flussab des Turbinenauslasses

Spüldotationen von $12 \mathrm{~m}^{3} / \mathrm{s}$, die vor der Hauptlaichzeit im Spätwinter sowie im Spätsommer vorgesehen sind, werden einerseits - zumindest in geringem Umfang - Sohlumlagerungen

und somit eine Aufbereitung von Kieslaichplätzen für lithophile Fischarten bewirken (Abb. 7). Andererseits werden damit Feinsedimentanlandungen in den Uferbereichen wieder ausge-

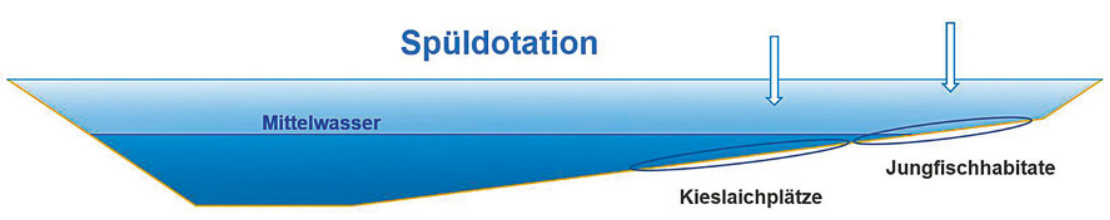

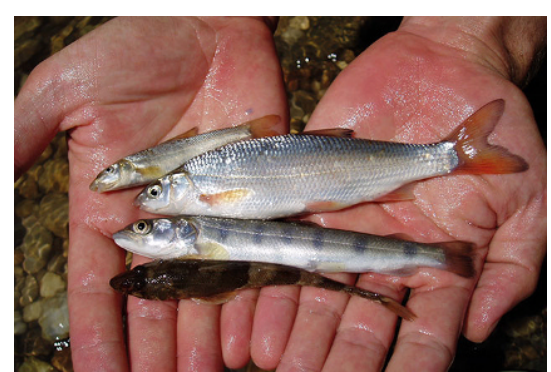

Abb. 8 Typische rheophile Fischartenvon oben nach unten: Barbe, Nase, Huchen und Streber. Letzterer gilt im gesamten Salzach-Inn-System als ausgestorben

tragen, die in einem glazial geprägten Gewässersystem wie am Inn verstärkt auftreten. Die Spüldotation soll somit wie die Hochwasserdynamik in einem natürlichen Fließgewässer - die nachhaltige Funktionalität der Gewässerlebensräume im Umgehungsgewässer gewährleisten. Sie ist insbesondere für den Erhalt der Qualität von Laichplätzen und Jungfischhabitaten essenziell. Ausgetragenes Geschiebe wird dem System durch Geschieberückführungen oder Geschiebebeigaben wieder zugeführt. Hierfür wurde im unteren Bereich des Umgehungsgewässers eine Geschiebefalle vorgesehen.

Gewässertypische Strukturen wie Furten, angeströmte Kiesbänke, Flachwasserzonen, Totholz und Buchten, die im gesamten Umgehungsgewässer errichtet wurden, bilden wertvolle Schlüsselhabitate insbesondere für Juvenilstadien rheophiler Fischarten (Abb. 8).

Das Umgehungsgewässer verläuft großteils parallel entlang des Stauhaltungsdamms in Form eines naturnahen, pendelnden Gerinnes mit einer Abfolge von Kolk-Furt-Sequenzen. Um das nachhaltige Bestehen von Kolken bzw. Tiefstellen im Gerinne zu gewährleisten, wurden Kurzbuhnen im Pralluferbereich, Langbuhnen an Gleituferbereichen und möglichst kompakte Raubaum- und Totholzstrukturen eingebaut (Abb. 9), welche Engstellen bzw. Zwangspunkte erzeugen. Dadurch werden bei hohen Abflüssen lokal hohe Schleppspannungen erzeugt, die Tiefstellen im Gerinne freihalten können.

Abb. 7 Regelprofil-WiederkehrendeSpüldotationen(12 $\left.\mathrm{m}^{3} / \mathrm{s}\right)$ gewährleisten die dauerhafte gewässerökologische Funktionalität der geschaffenen Lebensräume 


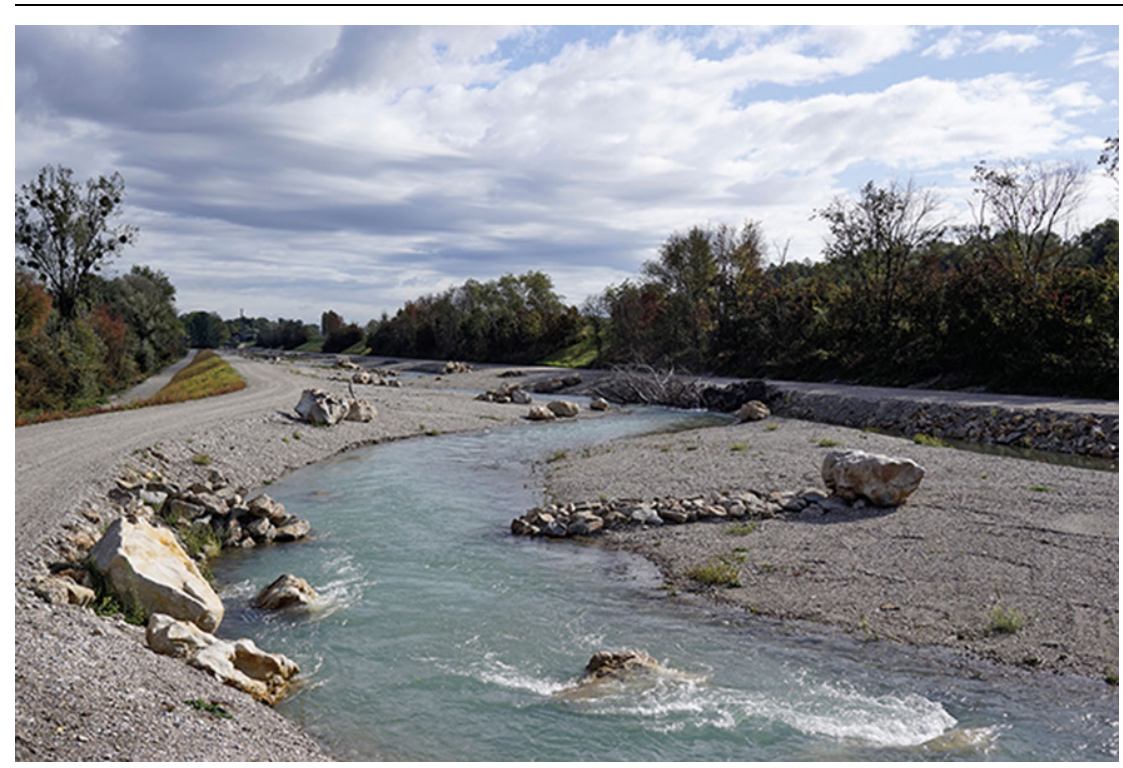

Abb. 9 Strukturierung des Umgehungsgewässers mittels Buhnen, Störsteinen und Totholz (Abfluss $3 \mathrm{~m}^{3} / \mathrm{s}$ )

\subsection{Vernetzung mit Nebengewässern und Dynamisierung der „fossilen $A u^{\prime \prime}$}

Seit Kraftwerkserrichtung sind die ausgedämmten Auen am Unteren Inn praktisch vollständig vom Hauptfluss entkoppelt. Im ursprünglichen Zustand traten dort jährlich Wasserspiegelschwankungen von mehreren Metern auf. Vor Maßnahmenumsetzung waren die natürlichen Schwankungen der Oberflächen- und Grundwasserstände auf ein Minimum reduziert.

Eine Redynamisierung dieser „fossilen $\mathrm{Au}$ " bzw. der Grund- und Altwasserwasserstände wird durch Anbindung eines Auegewässerkomplexes an das neu geschaffene Umgehungsgewässer erreicht (Abb. 5). Über drei Gräben mit verschließbaren Anbindungsbauwerken werden großflächig wieder autypische Wasserspiegelschwankungen von 0,8 m über sowie unter den Mittelwasserstand hergestellt. Zusätzlich wurden in den bereits stark verlandeten Gewässern Tiefstellen wiederhergestellt.

Eine Einwanderung von Fischen aus dem Inn in die „Hinterlandgewässer" (Augewässer hinter dem Stauhaltungsdamm) wird über das Umgehungsgewässer und die hergestellten Verbindungsgräben ermöglicht. Durch die Wasserstandsschwankungen wird neben den auökologischen Effekten auch die Qualität der Uferbereiche als Laichhabitat für phytophile Fischarten stark erhöht. Entsprechend dem
Flood-pulse-Konzept ist auch von einer Ankurbelung der Produktivität des Altwassersystems auszugehen (Junk et al. 1989).

\subsection{Insel-Nebenarmsystem}

Im unmittelbaren Unterwasser des Kraftwerks Ering-Frauenstein (Stauwurzel Kraftwerk Egglfing-Obernberg) wurde auf einer Eingriffsfläche von rund 30 ha großräumig Gewässerlebensraum in Form eines Insel-Nebenarmsystems und eines unterstromig angebundenen Altarms geschaffen (Abb. 10 und 11).
Solche Maßnahmen weisen in einer Staukette nur in den Stauwurzelbereichen eine hohe Funktionalität und Beständigkeit auf, weil hier noch annähernd natürliche Wasserspiegelschwankungen und Fließgeschwindigkeiten vorherrschen.

Unmittelbar flussab des Kraftwerks wurde das linke, bisher mit Blockwurf gesicherte Ufer auf einer Länge von $2,5 \mathrm{~km}$ zur Gänze rückgebaut und in ein flaches Ufer mit kontinuierlichem Wasser-Land-Übergang umgewandelt. Durch die Herstellung eines rund 1,5 km langen und $80 \mathrm{~m}$ breiten Nebenarms auf ehemaligen Auwald- und Ackerflächen ist eine große Flussinsel entstanden.

Durch Anlage eines breiten Einströmbereichs in den Nebenarm sowie eines flach ausgebildeten Inselkopfs entstanden großflächig Kiesstrukturen, die insbesondere bei Mittelwasserführung eine gute Eignung als Laichplätze aufweisen.

Die Verlandung mit Feinsedimenten kann speziell in Nebenarmsystemen zum Verlust von flachen Kiesufern führen (Zauner et al. 2016a). Wichtig ist daher, dass der Nebenarm entsprechend breit und abflussstark konzipiert ist. Der große hydraulische Radius führt dazu, dass die in den Uferbereichen wirkenden Sohlschubspannungen auch bei geringem Gefälle noch vergleichsweise hoch sind und Feinsedimentanlandungen hintangehalten werden. Ein Teil des Aushubs wurde in den Hauptarm des Inns als Kiesschüttung mit Feinsedimentkern vorgebaut. Dadurch konnten einerseits Flachwas-

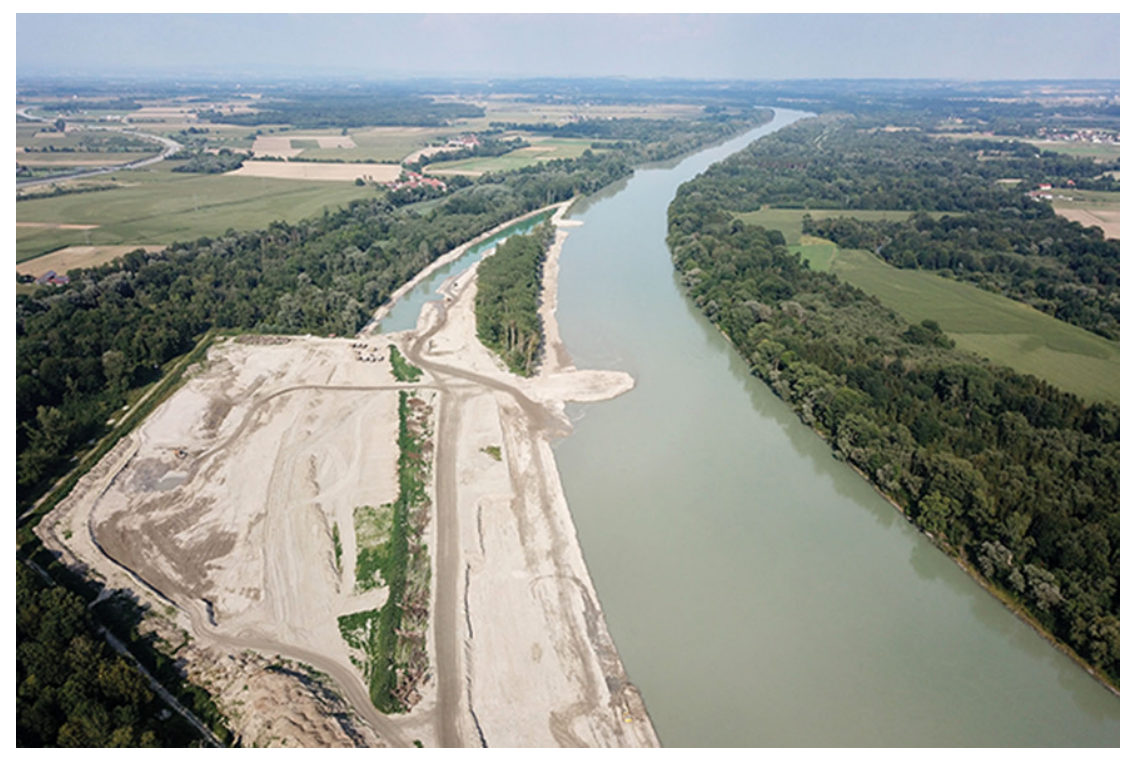

Abb. 10 Das Insel-Nebenarmsystem in der Bauphase 


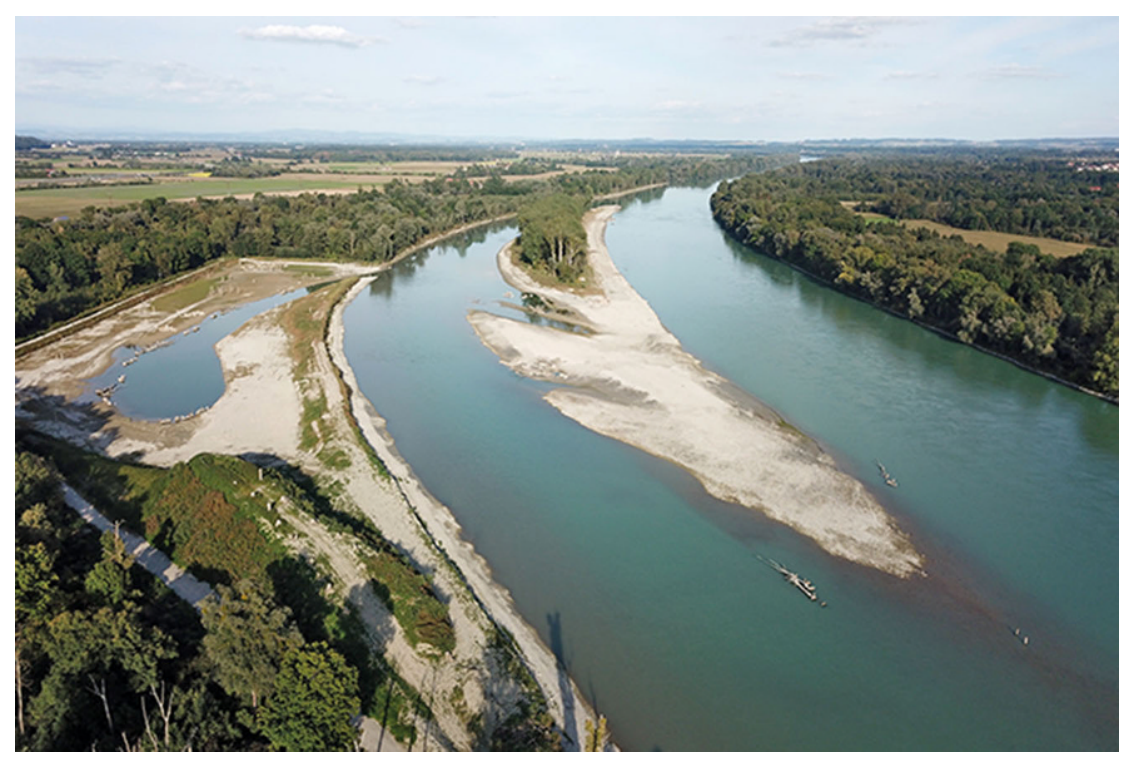

Abb. 11 Das fertig gestellte Insel-Nebenarmsystem bei Niederwasser nach Durchgang eines einjährlichen Hochwassers

serbereiche vergrößert und andererseits der Hauptfluss etwas eingeengt werden, was die Durchströmung des Nebenarms verbessert. Durch Strukturierung des Insel-Nebenarmsystems mittels Totholz im Nieder- bis Mittelwasserbereich können kleine Buchten als optimale Jungfischhabitate entstehen und dauerhaft erhalten werden.

Ein im Augebiet liegender Acker wurde flächig um etwa $2 \mathrm{~m}$ abgesenkt und für die Errichtung eines Stillgewässers mit einer Wasserfläche bei Mittelwasserführung von rund 1,7 ha genutzt (Abb. 11 und 12), welches unterstromig an den Nebenarm angebunden ist. Die Anbindung wurde bewusst schmal ausgeführt, um den Wasseraustausch mit dem Inn möglichst gering und somit die Verlandung der Stillgewässerstruktur mit Feinsedimenten hintanzuhalten.

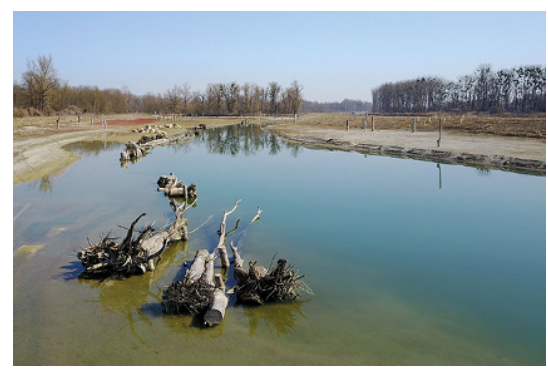

Abb. 12 Das neu errichtete, unterstromig an den Nebenarm angebundene Stillgewässer mit eingebauten Totholzstrukturen
Durch Geländeabsenkungen auf das Mittelwasserniveau ist das Entstehen großer Flächen mit Pioniervegetation zu erwarten, wodurch bei erhöhter Wasserführung eingestaute Vegetation als Laich- und Juvenilhabitat für phytophile Fischarten zur Verfügung steht. Tiefstellen bieten zudem hochwertige Winterhabitate, welche erfahrungsgemäß auch von rheophilen Fischarten angenommen werden. Zur Verbesserung und Diversifizierung des Lebensraums wurden Totholzstrukturen im Bereich der NiederwasseranschlagsliHauptfluss zugegeben nie sowie in tiefer liegenden Bereichen eingebaut.

Die abgesenkten Flächen unterliegen aufgrund der Lage am oberen Ende der Stauwurzel einer annähernd natürlichen Wasserstandsdynamik. Auf den über Mittelwasser liegenden Flächen können sich erstmals wieder inntypische Pioniergehölze entwickeln (z.B. Reifweide oder Lavendelweide).

Insgesamt wurde für die Herstellung der Maßnahmen am Innkraftwerk Ering-Frauenstein knapp eine Million $\mathrm{m}^{3}$ Sedimente umgelagert. Rund die Hälfte davon wurde für die Herstellung der Strukturierungsmaßnahmen und des Umgehungsgewässers wieder eingebaut. Flussbürtige Feinsedimente wurden zum Teil auch in den Inn vorgeschüttet, wo sie bei höheren Abflüssen rasch remobilisiert wurden (Abb. 13).

Das Projektgebiet befindet sich im Natura-2000-Gebiet DE7744371 „Salzach und Unterer Inn“. Durch die Maßnahmen ist eine deutliche Förderung zahlreicher Schutzgüter (z.B. FFHFischarten, Biber, Fischotter, Weichholzau) zu erwarten. Temporäre Verluste von FFH-Lebensraumtypen (z.B. Weichholzau) und von Habitaten für Arten wie Scharlachkäfer oder Fledermäuse mussten durch Begleitmaßnahmen ausgeglichen werden. So wurden vorübergehende Lebensraumengpässe vermieden, indem eine Reihe von Schutz- und Vermeidungsmaßnahmen (z.B. Totholzmanagement) sowie vorgezogene Ausgleichsmaßnahmen (z.B. Aufhängen von Nistkästen für Fleder-

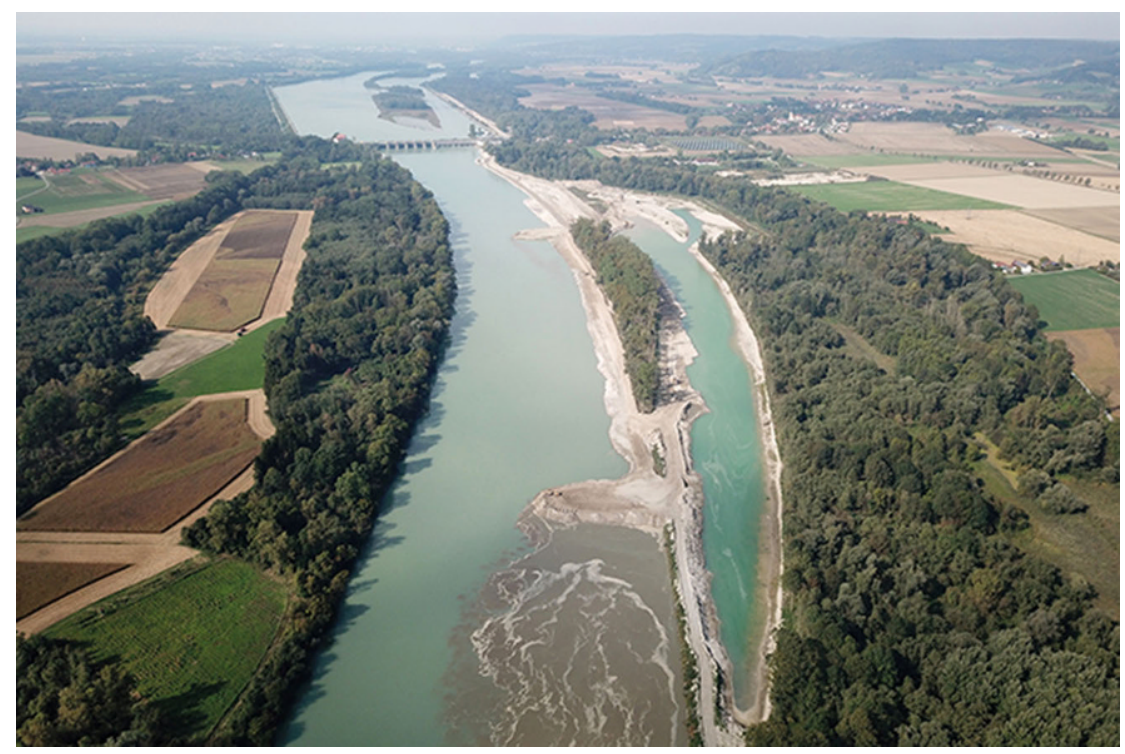

Abb. 13 Flussbürtige Sedimente wurden zum Teil in Form zweier Vorschüttungen dem 
mäuse und Vögel) umgesetzt wurden. Außerdem ist ein umfangreiches Monitoring der umgesetzten Maßnahmen vorgesehen.

\section{Fischökologische Ist-Situation}

\subsection{Hauptfluss}

Als Prämonitoring der beschriebenen Strukturierungsmaßnahmen wurden 2018 fischökologische Untersuchungen im Stauraum des Innkraftwerks Egglfing-Obernberg durchgeführt. Methodisch orientierten sich diese an den Erhebungen zum WRRL-Monitoring in großen Flüssen („D-Stellen“), d.h., es wurden Elektrobefischungen mittels Anodenrechen und Polstange am Tag und in der Nacht durchgeführt und als ergänzende Methoden kamen Multimaschennetze, Langleinen sowie das elektrische Bodenschleppnetz zum Einsatz. Erhebungen erfolgten in der Stauwurzel, im Übergangsbereich und im zentralen Stau zu je einem Termin Ende Juli/Anfang August und Mitte Oktober. Die Befischungen konzentrierten sich auf den Hauptfluss, Verlandungsbereiche mit Altarmcharakter im zentralen Stau wurden nur in geringem Umfang beprobt.

Die Ergebnisse dieser Befischungen bezüglich der Artverteilung sind in Abb. 14 dargestellt. Insgesamt wurden 4109 Individuen aus 35 verschiedenen Arten nachgewiesen. Die drei Arten
Laube, Aitel und Nase machten mit 2916 Individuen $71 \%$ des Gesamtfangs aus. Die nächsthäufigeren Arten waren Flussbarsch, Barbe, Dreistachliger Stichling, Kaulbarsch, Donau-Weißflossengründling, Hasel, Hecht und Schnei- der. Zu erwähnen ist der Nachweis von 29 Individuen des Ukrainischen Bachneunauges, einer Art, deren Larven (Querder) die Feinsedimentbänke im zentralen Stau besiedeln. Die in nennenswerten Stückzahlen nachgewie-

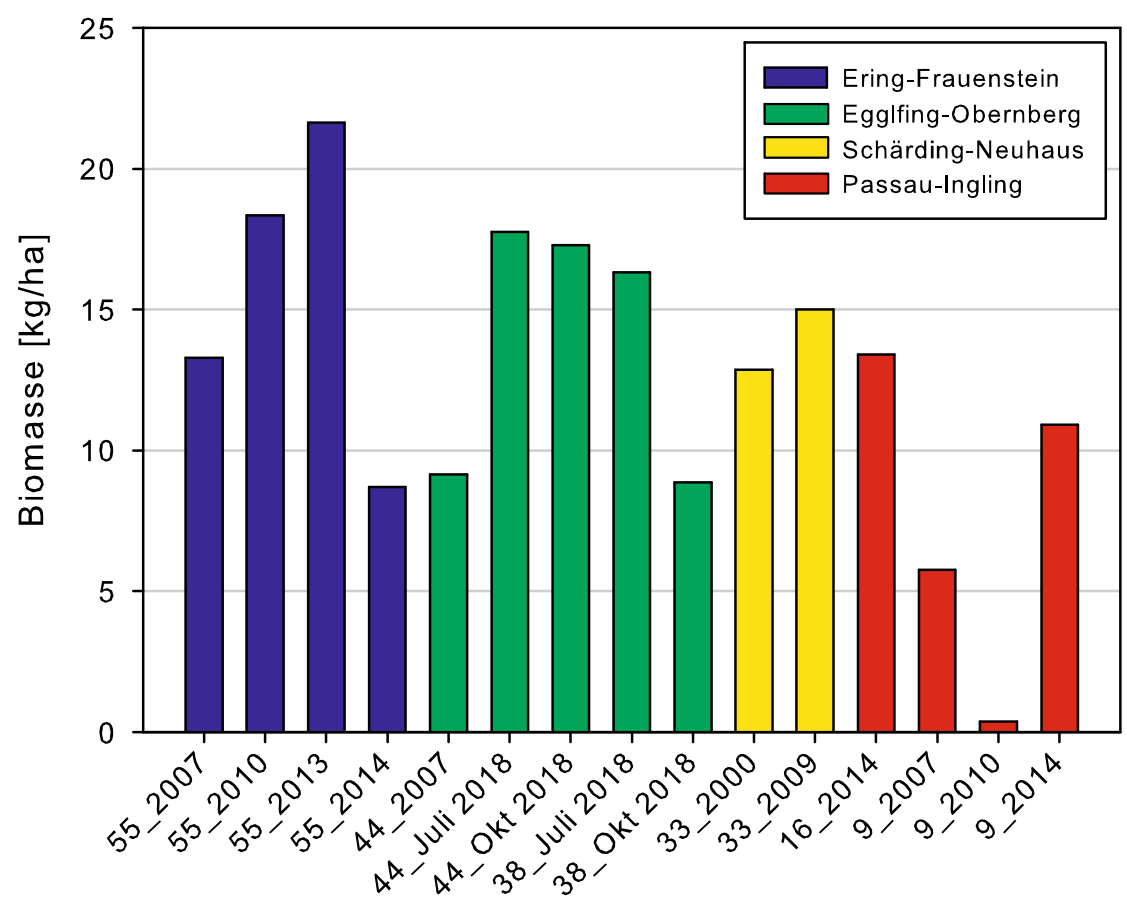

Fluss-km_Jahr

Abb. 15 Vergleich der Fischbiomasse bei quantitativen Erhebungen am Unteren Inn. Datenquellen: GZÜV, Zauner et al. (2001), Zauner et al. (2019)

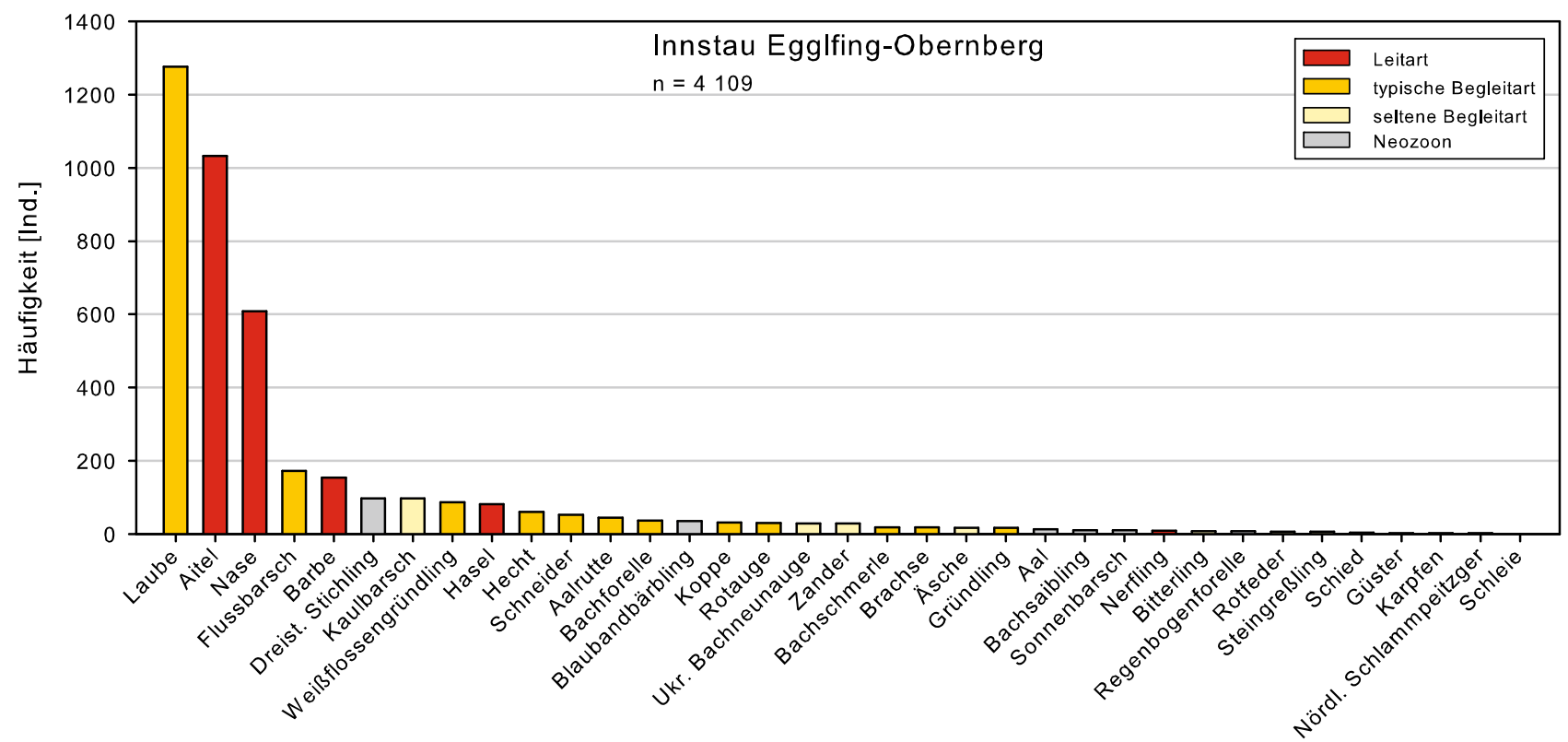

Abb. 14 Art-Rangkurve der 2018 im Inn gefangenen Fische (alle Methoden). Die Farbe gibt die Einstufung laut fischökologischem Leitbild wieder 
senen rhithralen Arten Koppe, Äsche, Bachforelle, Bachschmerle und Ukrainisches Bachneunauge unterstreichen das kühle Temperaturregime des Unteren Inns. Die typischen wärmeliebenden bzw. stagnophilen Arten Bitterling, Sonnenbarsch, Karpfen, Güster und Schleie wurden jeweils nur anhand weniger Individuen nachgewiesen. Diese Arten besiedeln primär die Nebengewässer und kommen im Hauptfluss auch im zentralen Stau nur in geringen Dichten vor. Der Steingreßling wurde erstmals für den Unteren Inn und erstmals seit ca. 150 Jahren für das gesamte Salzach-Inn-System nachgewiesen, wo er als verschollen galt (Jung et al. 2019). Der Nachweis zweier Individuen einer asiatischen Schlammpeitzgerart stellte den Erstnachweis für Österreich dar, während die Art entlang der rein deutschen Innstrecke stromauf des Untersuchungsgebiets bereits seit 2013 bekannt ist. Anhand einer molekularbiologischen Untersuchung konnte sie dem aus China stammenden Nördlichen Schlammpeitzger (Misgurnus bipartitus) zugeordnet werden (Zangl et al. 2020).

Die Befischungsergebnisse wurden für eine Bewertung des ökologischen Zustands mittels Fisch Index Austria (FIA) herangezogen. Insgesamt wurden vier Bewertungen durchgeführt, indem Stauwurzel und Stau sowie die Sommer- und Herbsttermine getrennt herangezogen wurden. Aufgrund der geringen Biomasse ergeben alle Bewertungen einen „schlechten fischökologischen Zustand“ (FIA-Wert 5,00). Die Biomassewerte lagen mit 8,8 bis $16,0 \mathrm{~kg} / \mathrm{ha}$ deutlich unter dem Grenzwert („k.o.-Kriterium“) für den „unbefriedigenden Zustand“ (25 kg/ha) bzw. sehr deutlich unter jenem für den „guten Zustand“ (50 kg/ha). Ohne Biomasse-k.o. würden sich FIA-Werte zwischen 2,56 und 3,04 ergeben, was einem „mäßigen Zustand“ entspricht. Erwartungsgemäß fielen die Bewertungen im zentralen Stau durchwegs etwas schlechter aus als in der Stauwurzel.

Die aktuellen Ergebnisse ähneln sehr stark jenen des offiziellen österreichischen WRRL-Monitorings am Unteren Inn. Insgesamt liegen sieben Erhebungen zwischen 2007 und 2014 vor. Mit Ausnahme von zwei Aufnahmen im Stauraum des Innkraftwerks Ering-Frauenstein mit einem „unbefriedigenden Zustand“ ergaben sämtliche Erhebungen einen „schlechten Zustand“. Ohne Biomasse-k.o. lag mit einer Ausnahme bei allen Aufnahmen wie bei den aktuellen Erhebungen - ein „mäßiger Zustand“ vor. Die Biomassewerte lagen bei allen in der bayerischoberösterreichischen Grenzstrecke des Unteren Inn bisher durchgeführten Erhebungen unter $25 \mathrm{~kg} / \mathrm{ha}$ (Abb. 15).

Analysiert man die aktuellen FIABewertungen im Detail, so zeigen sich folgende Defizite: Abgesehen von der geringen Biomasse, bedingt insbesondere durch den sehr geringen Bestand der Leitarten Nase, Barbe, Nerfling und Aitel, ist vor allem das vollständige Fehlen der Leitfischart Huchen für das schlechte Bewertungsergebnis verantwortlich. Ein weiteres Defizit stellen die fehlenden Nachweise zweier typischer Begleitarten (Strömer, Wels) dar, wobei letztere Art im Gebiet vorkommt, der Bestand im Vergleich zur Donau allerdings recht gering sein dürfte. Noch deutlich höher ist der Artenfehlbetrag bei den seltenen Begleitarten, allerdings werden diese für die Bewertung nur sehr gering gewichtet. Bezüglich des Fischregionsindex (Werte zwischen 5,9 und 6,2) ergeben sich kaum Abweichungen zur Referenzsituation $(6,0)$. Die Artenzusammensetzung in Hinblick auf ihre längszönotischen Verbreitungsschwerpunkte hat sich gegenüber der Referenzsituation demnach wenig verschoben. Es dominieren nach wie vor Leitarten des Epipotamals wie Aitel, Barbe und Nase neben der typischen Begleitart Laube den Bestand.

In Hinblick auf den Populationsaufbau sind sowohl bei den Leitarten als auch bei den typischen Begleitarten starke Defizite erkennbar. Bezüglich der Leitarten betrifft dies insbesondere Nerfling und Hasel sowie im Stau auch die Barbe. Aber auch bei den häufigeren Leitarten Aitel und Nase konnten kaum Adultfische nachgewiesen werden.

Zusammenfassend stellen somit der insgesamt ausgesprochen geringe Fischbestand, das Fehlen der Leitfischart Huchen sowie von Begleitarten und die unbefriedigende Altersstruktur zahlreicher Leit- und typischer Begleitarten die zentralen Defizite dar. Die Ergebnisse spiegeln die hydromorphologische Situation im Gebiet vor Umsetzung der oben beschriebenen Maßnahmen, nämlich das Fehlen entsprechender Habitate rheophiler Flussfischarten, treffend wider.

Im Gegensatz zur österreichischen Donau, wo abgesehen von den anadromen Acipenseriden das ursprüngliche Arteninventar praktisch in allen Ab- schnitten nach wie vor vorhanden ist, sind am Unteren Inn einige Arten $u$. a. aufgrund von Kontinuumsunterbrechungen ausgestorben. Im Stauraum Egglfing-Obernberg trifft dies beispielsweise auf die Donauperciden Schrätzer, Streber, Zingel und Donaukaulbarsch, den Sterlet sowie die „stark potamalen“ Cypriniden Zobel, Zope, Sichling und wahrscheinlich auch den Frauennerfling zu. Diese Arten kommen entweder nur mehr in der kurzen Mündungsstrecke bis zum Innkraftwerk Passau-Ingling vor, oder das zweite Innkraftwerk Schärding-Neuhaus bildet die Verbreitungsgrenze (Zauner et al. 2019). Der für die nächsten Jahre geplanten Herstellung der Durchgängigkeit aus der Donau kommt somit sowohl in qualitativer als auch quantitativer Hinsicht eine hohe Bedeutung zu.

\subsection{Augewässer}

Im Zuge der Errichtung des Umgehungsgewässers wurden 2015 und 2019 Befischungen in den Altwässern der Eringer $\mathrm{Au}$ durchgeführt. Dabei wurden die Uferlinien vom Boot aus mittels Polstange befischt. Die fischökologische Situation vor Maßnahmenumsetzung soll hier kurz beschrieben werden.

In den beiden zusammenhängenden Augewässern konnte eine typische Zönose isolierter Altarme (Plesio- bzw. Paläopotamon) mit ausschließlich strömungsindifferenten und stagnophilen Arten nachgewiesen werden (Abb. 16 und 17). Die Artenzahl war mit 16 Arten recht gering. Es dominierten Rotauge, Moderlieschen, Rotfeder, Hecht, Schleie, Bitterling, Sonnenbarsch und Flussbarsch. All diese Arten reproduzieren in stagnierenden Habitaten und können somit ihren gesamten Lebenszyklus im Altwasser absolvieren. Typische Vernetzungszeiger konnten hingegen nicht (z.B. Schied, Nerfling,

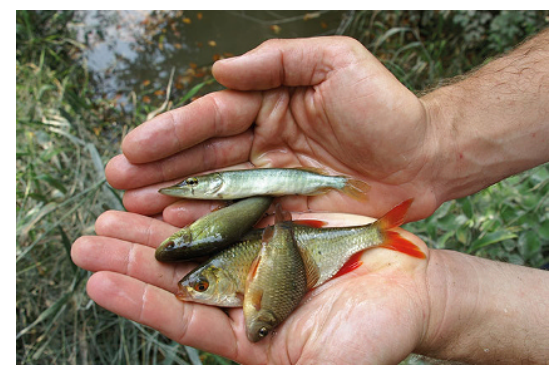

Abb. 16 Typische Fischzönose isolierter Augewässer: Hecht, Schleie, Rotfeder und Karausche 


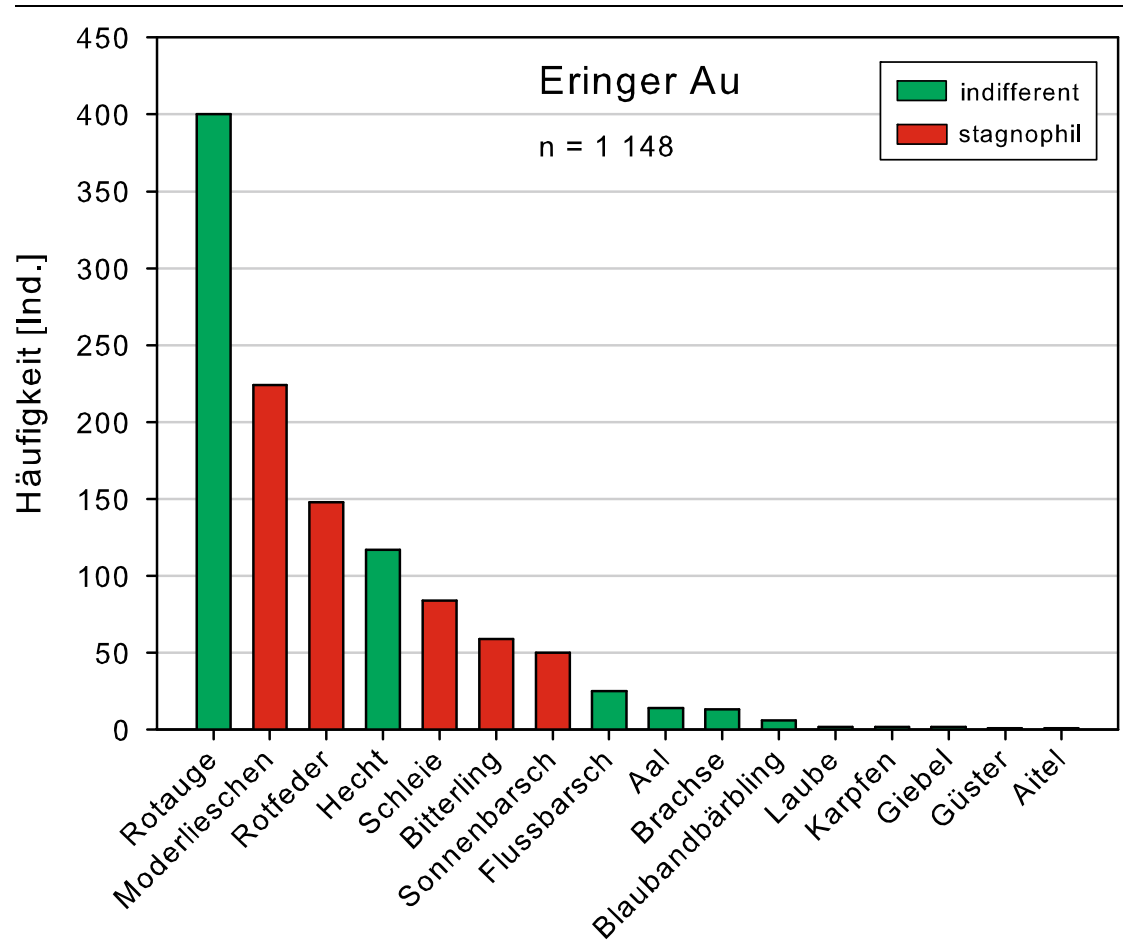

Abb. 17 Art-Rangkurve der insgesamt (2015 und 2019) in den Altwässern der Eringer Augefangenen Fische

Rußnase) oder nur in geringen Dichten (Aitel, Laube, Brachse, Güster) nachgewiesen werden, rheophile Arten fehlten gänzlich.

Insgesamt zeigt die Fischzönose die Isolation des Altwassers vom Hauptfluss treffend an. Grundsätzlich weist auch diese Zönose mit dem Moderlieschen als stark gefährdete Art sowie dem Bitterling als FFH-Art eine hohe naturschutzfachliche Wertigkeit auf. Isolierte Augewässer mit ähnlicher Fischzönose sind im Gebiet allerdings vergleichsweise zahlreich vorhanden, wohingegen unterstromig angebundene Altwässer mit natürlichen Wasserstandsschwankungen vor Maßnahmenumsetzung vollständig fehlten. Durch die Anbindung des Augewässers an das Umgehungsgewässer sowie durch Schaffung eines neuen angebundenen Altarms wurden diese Schlüsselhabitate für an laterale Konnektivität angewiesene Fischarten in hoher Qualität zur Verfügung gestellt. Es ist davon auszugehen, dass diese Maßnahmen auch zur Sanierung des Defizits bezüglich der Fischbiomasse im Hauptfluss und somit zur Erreichung des „guten ökologischen Potenzials“ beitragen.

\section{Erste Monitoringergebnisse der umgesetzten Maßnahmen}

Erste Monitoringergebnisse des Umgehungsgewässers am Innkraftwerk Ering-Frauenstein liegen in Bezug auf die Durchgängigkeit vor (Ratschan et al. 2020). So wurde im Herbst 2019 unmittelbar nach Flutung des Gerinnes ein knapp zwei Monate dauerndes Monitoring mittels „Ausstiegsreuse“ (Abb. 18) durchgeführt. Die Reuse war vom 25.9. bis 19.11.2019 insgesamt 55 Tage in Betrieb. In diesem Zeitraum konnten insgesamt 26.712 Individuen aus 25 verschiedenen Arten gefangen werden. Die durchschnittliche Aufstiegszahl lag somit bei 486 Individuen pro Tag.

Die Art-Rangkurve der nachgewiesenen Arten bietet einen Überblick über die Zusammensetzung des Gesamtfangs in der Reuse (Abb. 19). Wie bei den Bestandserhebungen im Hauptfluss stellte die Laube mit 16.422 dokumentierten Individuen den Hauptteil des Gesamtfangs. Die nächsthäufigen Arten waren Rotauge (3700 Ind.), Hasel (1858 Ind.) Brachse (1648 Ind.) und Nase (1629 Ind.). Bemerkenswert ist die hohe Anzahl von 93 aufgestiegenen Schieden, einer im Gebiet vergleichsweise selten vorkommenden (siehe Abb. 14) und bisher nur unregelmäßig

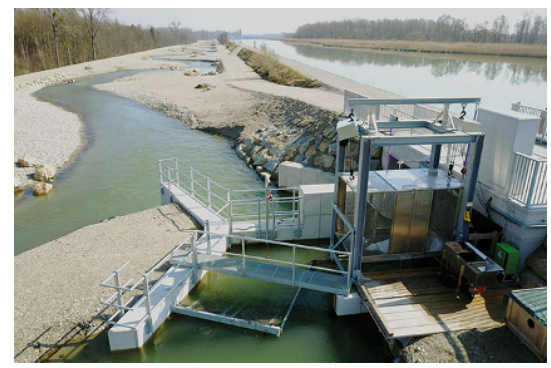

Abb. 18 Reusenanlage im Ausstiegsbereich des Umgehungsgewässers bei ca. $4 \mathrm{~m}^{3} / \mathrm{s}$ Abfluss und gehobenem Reusenkasten

im Zuge von Migrationsuntersuchungen dokumentierten FFH-Art.

Das Größenspektrum der gefangenen Fische reichte von Cypriniden mit unter $50 \mathrm{~mm}$ bis hin zu Hechten mit $680 \mathrm{~mm}$ Totallänge. Der noch fehlende Nachweis der größenbestimmenden Fischart (Wels mit $1200 \mathrm{~mm}$ ) überrascht insofern nicht, als der Wels im Unterwasser ausgesprochen selten vorkommt. Abgesehen von der Laube (Abb. 20) dominierten bei den einzelnen Cyprinidenarten durchwegs Subadulte. Diese führen im Herbst sehr ausgeprägte Wanderbewegungen durch, was auch im Rahmen anderer Migrationsuntersuchungen festgestellt werden konnte (z. B. Eberstaller et al. 2001).

Die festgestellten Aufstiegszahlen sind in Anbetracht des geringen Fischbestands im Unterwasser überraschend hoch und liegen deutlich über jenen von vergleichbaren Erhebungen an Fischaufstiegshilfen der Donau. So lag beispielsweise die durchschnittliche herbstliche Aufstiegszahl im Umgehungsgewässer am Donaukraftwerk Ottensheim-Wilhering bei 36 Individuen pro Tag (Zauner et al. 2017). Eine plausible Erklärung für den intensiven Fischaufstieg könnte ein sogenannter „opening effect“ sein, weil die Anlage erst 15 Tage zuvor geflutet worden war. Weiters fiel die Untersuchung offensichtlich zeitlich mit dem Beginn starker herbstlicher Wanderungen zusammen. Sehr wahrscheinlich werden diese Effekte durch eine günstige Auffindbarkeit und Durchwanderbarkeit des abflussstarken und im gesamten Lauf naturnah ausgeführten Umgehungsgewässers begünstigt. Im Jahr 2020 wird die eigentliche fischökologische Funktionskontrolle im Regelbetrieb durchgeführt, also mit der jahreszeitlich gestaffelten Dotation. Die Ergebnisse im Herbst 2020 werden zei- 


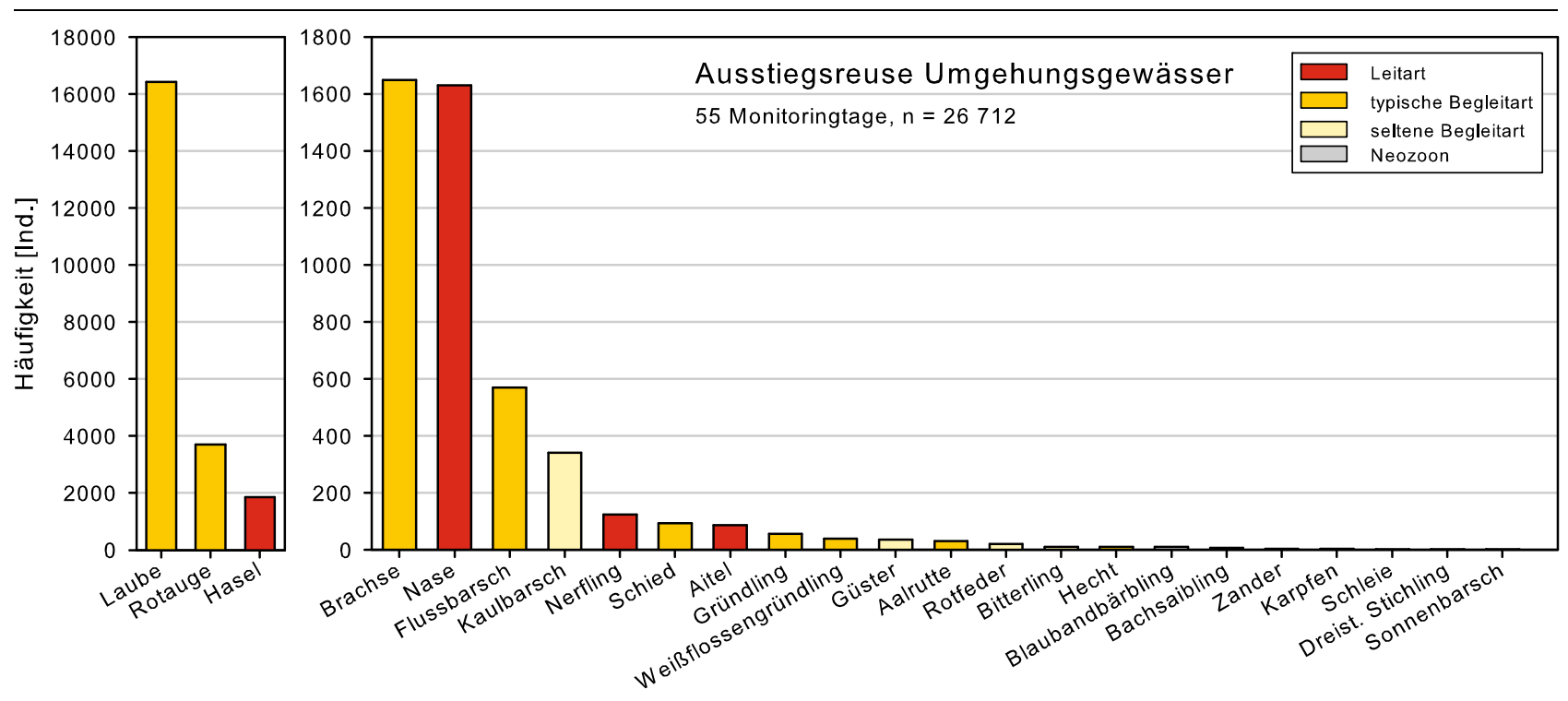

Abb. 19 Art-Rangkurve der in der Ausstiegsreuse im Rahmen des Pilotmonitorings gefangenen Fische $(n=26.712)$

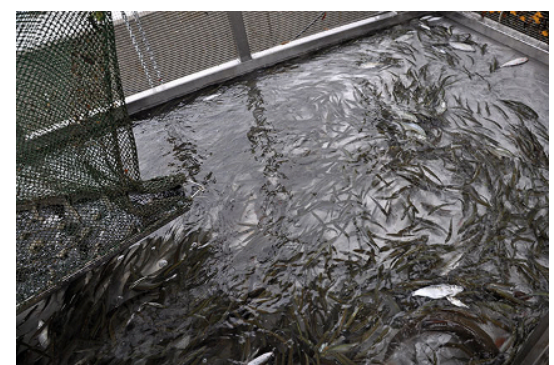

Abb. 20 Gehobener Reusenkasten mit an einem Herbsttag gefangenen Fischen

gen, ob es sich beim intensiven herbstlichen Aufstieg um ein wiederkehrendes Phänomen handelt. Die Auffindbarkeit und die Funktion als Laichhabitat auch für anspruchsvolle Fischarten wird durch ein am 19.4.2020 aufgenommenes Amateurvideo bestätigt, das zwei große, laichbereite Huchen im oberen Teil des Umgehungsgewässers zeigt (https://www.youtube.com/ watch?v=pRoUhvLbpxI).

\section{Conclusio und Ausblick}

Über die in Kap. 2. beschriebene, auf Fischlebensräume fokussierende Herleitung des „guten ökologischen Potenzials" hinaus wurden von Koller-Kreimel (2011) sowie im aktuellen „HMWBLeitfaden“ (Eberstaller et al. 2015) für konkrete Belastungskombinationen erheblich veränderter Wasserkörper auch biologische Definitionen vorgegeben. Als Richtwert in einem staubeeinflussten Fließgewässer gelten dazu in der Stauwurzel die Werte des „Fisch Index
Austria“ für den „guten ökologischen Zustand" (Note $\leq 2,50$ ) inklusive einer ausreichenden Biomasse. Ergänzend sind im zentralen Stau Maßnahmen mit hoher ökologischer Wirksamkeit umzusetzen.

Dieser Zugang mit einer Fokussierung auf die Stauwurzel ist sowohl in Hinblick auf den Leitgedanken der Wasserrahmenrichtlinie nachvollziehbar, sich am Leitbild des anthropogen unbeeinträchtigten Gewässers zu orientieren, als auch vor dem Hintergrund, dass Stauwurzeln in aller Regel wesentlich günstigere Rahmenbedingungen für nachhaltig wirksame, kosteneffiziente und multifunktionale Maßnahmen bieten als stärker staubeeinflusste Abschnitte. Diverse Untersuchungen im Rahmen von Renaturierungsprojekten an der Donau haben gezeigt, dass hydromorphologische Defizite in Stauketten großer Fließgewässer mittels am Leitbild orientierter Maßnahmen durchaus effektiv saniert werden können (z. B. Jungwirth et al. 2005; Schmutz et al. 2013; Zauner et al. 2015b; Meulenbroek et al. 2018; Waidbacher et al. 2018). So war beispielsweise im Donaustauraum Aschach im Zeitraum 1989 bis 2008 nach Umsetzung von Maßnahmen in der Stauwurzel eine deutliche Erholung des ursprünglich sehr geringen Bestands der Leitfischart Nase mit einer Erhöhung der Biomasse auf das 15 bis 20-Fache zu verzeichnen (Zauner et al. 2016b). Aktuell laufende Erhebungen bestätigen diesen positiven Aufwärtstrend (Zauner und Jung in prep.). Die oben beschriebe- nen Maßnahmen in der Stauwurzel des Innkraftwerks Egglfing-Obernberg lassen ähnlich positive Entwicklungen für diesen Innstauraum erwarten. Allerdings dauert es in Potamalgewässern aufgrund der langen Generationsdauer der Leitarten viele Jahre, bis die volle Wirksamkeit der umgesetzten Maßnahmen insbesondere in Hinblick auf die Biomasse erkennbar wird.

In Abstimmung und mit Unterstützung der Naturschutzbehörden in Niederbayern und Oberösterreich hat Innwerk AG das LIFE-Projekt „Riverscape Lower Inn“ konzipiert, das darauf abzielt, mit einem systemischen, großräumigen Ansatz den ökologischen Wert des Gebiets langfristig zu sichern, und dessen Förderung im Februar 2020 beantragt. Neben der Errichtung eines dynamisch dotierten Umgehungsgewässers am Innkraftwerk EgglfingObernberg sowie einer großräumigen Strukturierung des Kraftwerksunterwassers sind Entlandungsmaßnahmen in Seitenbereichen des Stauraums Egglfing-Obernberg zur Wiederherstellung von Gewässerlebensraum sowie Verbesserungen an den Mündungen der Zubringer Stampfbach und Mühlheimer Ache wesentliche Bestandteile des beantragten LIFE-Projekts. Die Umsetzung der geplanten Maßnahmen stellt einen wichtigen Beitrag zur Erreichung des "guten ökologischen Potenzials“ in den Detailwasserkörpern des Grenzinns dar.

Open Access Dieser Artikel wird unter der Creative Commons Namensnen- 
nung 4.0 International Lizenz veröffentlicht, welche die Nutzung, Vervielfältigung, Bearbeitung, Verbreitung und Wiedergabe in jeglichem Medium und Format erlaubt, sofern Sie den/die ursprünglichen Autor(en) und die Quelle ordnungsgemäß nennen, einen Link zur Creative Commons Lizenz beifügen und angeben, ob Änderungen vorgenommen wurden.
Die in diesem Artikel enthaltenen Bilder und sonstiges Drittmaterial unterliegen ebenfalls der genannten Creative Commons Lizenz, sofern sich aus der Abbildungslegende nichts anderes ergibt. Sofern das betreffende Material nicht unter der genannten Creative Commons Lizenz steht und die betreffende Handlung nicht nach gesetzlichen
Vorschriften erlaubt ist, ist für die oben aufgeführten Weiterverwendungen des Materials die Einwilligung des jeweiligen Rechteinhabers einzuholen.

Weitere Details zur Lizenz entnehmen Sie bitte der Lizenzinformation auf http://creativecommons.org/licenses/ by/4.0/deed.de.
BMLFUW (2017): Nationaler Gewässerbewirtschaftungsplan 2015. Bundesministerium für Land- und Forstwirtschaft, Umwelt und Wasserwirtschaft, Sektion IV Wasserwirtschaft. Wien

Eberstaller, J., Köck, J., Haunschmid, R., Jagsch, A., Ratschan, C., Zauner, G. (2015): Leitfaden zur Bewertung erheblich veränderter Gewässer Biologische Definition des guten ökologischen Potentials. Version 02, Jänner 2015. BMLFUW. Wien

Eberstaller, J., Pinka, P., Honsowitz, H. (2001): Überprüfung der Funktionsfähigkeit der FAH am KW Freudenau. Schriftenreihe der Forschung im Verbund 72: 1-95

Jung, M., Ratschan, C., Zauner, G. (2019): Erstnachweis des Steingreßlings (Romanogobio uranoscopus Agassiz, 1828) im Inn und Verbreitung im deutschsprachigen Raum. Österreichs Fischerei 72 (7): 177-189

Jungwirth, M., Haidvogl, G., Hohensinner, S. Muhar, S., Schmutz, S., Waidbacher, H. (2005): Leitbild-specific measures for the rehabilitation of the heavily modified Austrian Danube River. Large Rivers Vol. 15 (1-4): 17-36

Junk, W., Bayley, P., Sparks, R. (1989): The flood pulse concept in river-floodplain systems. In Dodge, D. (ed): Proceedings of the International Large River Symposium. Canadian Special Publications of Fisheries and Aquatic 106: 110-127 Koller-Kreimel, V. (2011): Leitfaden zur Bewertung erheblich veränderter Gewässer - Definition des guten ökologischen Potentials. ÖWAVSeminar „Qualitätszielverordnung Ökologie und Chemie - Oberflächengewässer“. Vorstellung und erste Erfahrungen aus der Umsetzung. Wien. 3. Feb. 2011

Meulenbroek, P., Drexler, S., Nagel, C., Geistler, M., Waidbacher, H. (2018): The importance of a constructed near-nature-like Danube fish by-pass as a lifecycle fish habitat for spawning nurseries, growing and feeding: a long-term view with remarks on management. Marine and Freshwater Research 69: 1857-1869

Mühlbauer, M. Zauner, G, Herrmann, T, Ratschan, C. (2011): Ökologisches Restrukturierungspotential der Innstufen an der Grenzstrecke zwischen Österreich und Deutschland. Studie im Auftrag der ÖBK und EON

Ratschan, C., Schöfbenker, M., Lauber, W. Zauner, G. (2020): Fischökologisches Pilotmonitoring des Umgehungsgewässers EringFrauenstein. Studie im Auftrag der Innwerk AG

Schmutz, S., Kremser, H., Melcher, A., Jungwirth, M., Muhar, S., Waidbacher, H., Zauner, G. (2013): Ecological effects of rehabilitation measures at the Austrian Danube: a meta-analysis of fish assemblages. Hydrobiologia 729: 49-60. DO https://doi.org/10.1007/s10750-013-1511-z

Waidbacher, H., Drexler, S.-S., Meulenbroek, P. (2018): Danube Under Pressure: Hydropower Rules the Fish, in: Schmutz, S., Sendzimir, J. (Eds.): Riverine Ecosystem Management. Springer, Cham, S. 473-489

Zangl, L., Jung, M., Gessl, W., Koblmüller, S. Ratschan, C. (2020): Oriental or not: First record of an alien weatherfish (Misgurnus) species in Austria verified by molecular data. BioInvasion Records 9 (in press). https://www.reabic.net/ journals/bir/2020/Accepted.aspx

Zauner, G., Jung, M. (in prep.): Fischökologisches Monitoring der Kompensationsbaggerungen im Bereich Schlögen (Donaustauraum Kraftwerk Aschach). Studie im Auftrag der VERBUND Hydro Power GmbH

Zauner, G., Glatzel, J., Pinka, P. (2001): NaturProjekt „Unterer Inn mit Auen“. Fischbiologische Untersuchung Reichersberger Au und fischereiliches Bewirtschaftungskonzept. Studie im Auftrag des Amtes der Oö. Landesregierung
Zauner, G., Mühlbauer, M., Herrmann, T., Lauber, W., Ratschan, C. (2015a): Erreichung des guten ökologischen Potentials am Inn vom KW Braunau-Simbach bis zum KW Egglfing-Obernberg. Studie im Auftrag der Innwerk AG Zauner, G., Jung, M., Mühlbauer, M., Ratschan, C. (2015b): Fischökologische Sanierung von Fließstrecken und Stauhaltungen der österreichischen Donau gem. WRRL: Immer der Nase (Chondrostoma nasus) nach. Österreichs Fischerei 68 (7): 177-196

Zauner, G., Jung, M., Ratschan, C. (2016a): Fischökologisches Monitoring der Donau-Nebenarme Marktau (Wilhering) und Reischelau (Machland). Studie im Auftrag der Oö. Landesregierung, Direktion Umwelt und Wasserwirtschaft, Abt. Oberflächengewässerwirtschaft

Zauner, G., Jung, M., Ratschan, C., Mühlbauer, M. (2016b): Ökologische Sanierung von Fließstrecken und Stauhaltungen der österreichischen Donau - auf dem Weg zur Zielerreichung nach Wasserrahmenrichtlinie. Österreichs Wasserwirtschaft 68 (11-12):503-518

Zauner, G., Jung, M., Lauber, W., Mühlbauer, M., Ratschan, C. (2017): Dynamischer Umgehungsarm Donaukraftwerk Ottensheim-Wilhering Durchgängigkeit und Lebensraum. Wasserwirtschaft 12: 45-51

Zauner, G., Jung, M., Ratschan, C., Schöfbenker, M. (2019): Erhebung der Fischzönose im Innstauraum KW Egglfing-Obernberg. Fischökologische Situation vor Maßnahmenumsetzung. Studie im Auftrag der Innwerk AG

Hinweis des Verlags Der Verlag bleibt in Hinblick auf geografische Zuordnungen und Gebietsbezeichnungen in veröffentlichten Karten und Institutsadressen neutral. 